\title{
A Flux Splitting Scheme With High-Resolution and Robustness for Discontinuities
}

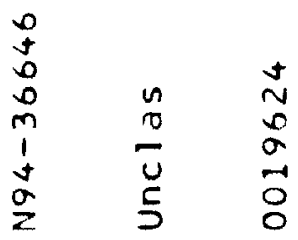

Yasuhiro Wada

Institute for Computational Mechanics in Propulsion

Lewis Research Center

Cleveland, Ohio

and National Aerospace Laboratory

Tokyo, Japan

Meng-Sing Liou

National Aeronautics and Space Administration

Lewis Research Center

Cleveland, Ohio

Prepared for the

32nd Aerospace Sciences Meeting and Exhibit

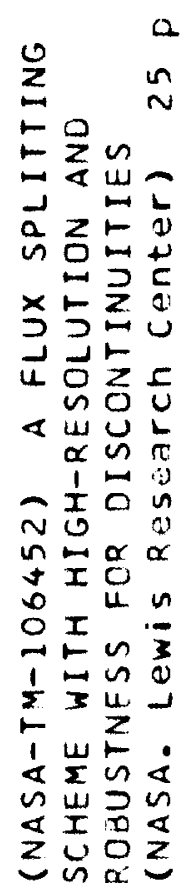

sponsored by the American Institute of Aeronautics and Astronautics Reno, Nevada, January 10-13, 1994

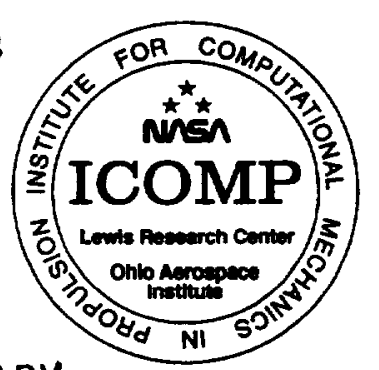





\title{
A Flux Splitting Scheme With High-Resolution and Robustness for Discontinuities
}

\author{
Yasuhiro Wada \\ Institute for Computational Mechanics in Propulsion \\ NASA Lewis Research Center, Cleveland, Ohio 44135 \\ and National Aerospace Laboratory, Tokyo, Japan \\ Meng-Sing Liou \\ National Aeronautics and Space Administration \\ Lewis Research Center, Cleveland, Ohio 44135
}

\begin{abstract}
A flux splitting scheme is proposed for the general non-equilibrium flow equations with an aim at removing numerical dissipation of Van-Leer-type flux-vector splittings on a contact discontinuity. The scheme obtained is also recognized as an improved Advection Upwind Splitting Method(AUSM) where a slight numerical overshoot immediately behind the shock is eliminated. The proposed scheme has favorable properties: high-resolution for contact discontinuities; conservation of enthalpy for steady flows; numerical efficiency; applicability to chemically reacting flows. In fact, for a single contact discontinuity, even if it is moving, this scheme gives the numerical flux of the exact solution of the Riemann problem. Various numerical experiments including that of a thermo-chemical nonequilibrium flow were performed, which indicate no oscillation and robustness of the scheme for shock/expansion waves. A cure for carbuncle phenomenon is discussed as well.
\end{abstract}

\section{INTRODUCTION}

Recently various high-resolution schemes have been devised for the Euler/Navier-Stokes equations[1-9]. Most of these schemes make use of a first-order upwind differencing as a basis, achieving higher-order accuracy under some restriction such as Total Variation Diminishing(TVD). Since these high-resolution schemes inherit their characteristics from their basic schemes, it is very important to design a basic scheme with desirable properties. Up to now several basic upwind schemes have been proposed, and most of them are categorized as either Flux Difference Splitting(FDS) or Flux Vector Splitting(FVS). The former uses an approximate solution of the local Riemann problem, while the latter splits the flux vector into up-stream and down-stream traveling components.

The most rigorous approach of the FDS scheme may be the use of the exact Riemann solver, as proposed by Godunov[10]. Since the Riemann problem is a nonlinear problem, this scheme requires iterative numerical procedures, leading to an inefficient method especially for vector machines or for gases with a general equation of state. Because all that is needed from the Riemann solution is a flux across a cell interface, some approximations should be possible. The most successful and popular approximate Riemann solver is Roe's method, which is the exact solution of a linearized Riemann problem[11]. This is able to capture any single stationary discontinuity with no numerical dissipation, unfortunately including an expansion shock. Such an unphysical solution can be removed by "Entropy Fix[5]." At strong expansions, however, the Roe scheme diverges even if the entropy fix is applied. In addition, it is known that the Roe scheme has a more serious problem, so-called "carbuncle phenomenon," which is a numerical instability in capturing a strong shock wave in multi-dimensional computation. Recently Quirk[12] has reported that even the exact Riemann solver suffers from this problem. Roe's approximate Riemann solver has consistency with the exact one about the conservation law, and this type of approximate Riemann solvers are called "Godunov-type" schemes[13]. Another Godunov-type scheme is the HLLE scheme, which much simplifies the Riemann problem by expressing the solution with only two waves. Einfeldt $[14,15]$ has shown that this scheme satisfies the positivity and entropy conditions if some appropriate velocities are chosen for these two waves. The HLLE scheme, however, lacks the information of contact discontinuity, so it is too dissipative to be applied to the Navier-Stokes equations although that dissipation is strong enough to remove the carbuncle phenomenon[12]. The HLLE scheme can be modified to include the information of the contact discontinuity under the positivity condition[16]. However, that scheme again suffers from the carbuncle phenomenon[17]. Also numerical noise radiated from slowly-moving shock waves has been reported for the 
exact Riemann solver as well as the Roe scheme[18], and is probably a common problem for any Godunovtype schemes. Another approximate Riemann solver was devised by Osher[19]. This scheme is not rigorously a Godunov-type scheme, but it captures a single shock wave at most by two-intermediates points, and reproduces the exact solution if a single expansion wave or contact discontinuity is present. Further, Osher's scheme does not produce the noise at slowly-moving shock waves. However, it fails in near vacuum conditions because it uses the reversed-order integration path in phase space, which is needed to avoid a serious overshoot in capturing shock waves.

In FVS, the flux function $\mathbf{F}$ is divided into positive and negative parts:

$$
\mathbf{F}(\mathbf{q})=\mathbf{F}(\mathbf{q})^{-}+\mathbf{F}(\mathbf{q})^{+}
$$

which give the numerical flux at cell interface between the states $q_{I}$ and $q_{R}$ :

$$
\mathbf{F}_{1 / 2}\left(\mathbf{q}_{L}, \mathbf{q}_{R}\right)=\mathbf{F}\left(\mathbf{q}_{L}\right)^{+}+\mathbf{F}\left(\mathbf{q}_{R}\right)^{-} \text {. }
$$

Steger and Warming[20] made use of similarity transformations and the homogeneity property of the Euler equations, splitting the flux depending on the sign of eigenvalues of the flux Jacobian matrix. This splitting showed errors around the sonic point, and Van Leer[21] proposed an alternative splitting, which gives noticeably better results and produces steady shock profiles with at most two zones. Hänel[22] further modified Van Leer's FVS so as to preserve total enthalpy for the steady state solution. These FVS schemes, however, share a serious disadvantage, that is, large dissipation on contact discontinuities and shear layers. Liou and Steffen[23] tried to remove the dissipation by using higher-order polynomial expansions of the flux vector, but their scheme was not robust enough in multi-dimensional calculations.

In another effort to develop less-dissipative upwind schemes, the surplus dissipation of the FVS is reduced by introducing the flavor of FDS into FVS schemes. These schemes are not FVS any more because their numerical flux can not be expressed in a form of Eq.(2), and we call them FV/DS. Hānel[24] has found that the numerical dissipation in the boundary layer is greatly reduced by using one-sided upwinding of the tangential velocity in Van Leer's FVS formulation. This was later extended by Van Leer[25], who employed one-sided upwinding also for the enthalpy of the energy fiux. But their schemes still hold the numerical dissipation for 1-D contact discontinuities and yield glitches in the pressure near the edge of the boundary layer. Liou and Steffen proposed a more promising scheme named Advection Upstream Splitting Method(AUSM)[26, 27], in which the cell-face advection Mach number is appropriately defined to determine the upwind extrapolation for the convective quantities. The AUSM can capture a stationary contact discontinuity with no numerical dissipation and is robust enough to calculate strong shock waves. However, it bears a slight numerical overshoot immediately behind the shock. Roe's assertion in 1988[28], "The perfect Riemann solver(including FVS) for generating numerical schemes does not yet exist." seems still true.

In this paper, we studied another way to remove the numerical dissipation of the Van-Leer-type flux-vector splittings on a contact discontinuity. The scheme obtained is also recognized as an improved AUSM scheme. Various numerical experiments, including a thermochemical nonequilibrium flow problem, are performed. A cure for the carbuncle phenomenon is discussed as well. Throughout this study we basically concentrate on the explicit first-order scheme in space and time in order to remove any uncertain factors other than the numerical flux itself.

\section{GOVERNING EQUATIONS}

2.1 Generalized Nonequilibrium Flow Equations Generally, a nonequilibrium flow such as described by chemical reactions or turbulent models has the governing equations in a form:

$$
\frac{\partial \mathbf{q}}{\partial t}+\frac{\partial \mathbf{F}_{k}}{\partial x_{k}}=\mathbf{S}
$$

where

$$
\begin{gathered}
\mathbf{q}=\left[\begin{array}{c}
\rho \\
\rho u_{1} \\
\rho u_{2} \\
\rho u_{3} \\
E \\
\rho f_{1} \\
\rho f_{2} \\
\vdots \\
\rho f_{n}
\end{array}\right], \quad \mathbf{F}_{k}=\left[\begin{array}{c}
\rho u_{k} \\
\rho u_{1} u_{k}+\delta_{1, k} p \\
\rho u_{2} u_{k}+\delta_{2, k} p \\
\rho u_{3} u_{k}+\delta_{3, k} p \\
(E+p) u_{k} \\
\rho f_{1} u_{k} \\
\rho f_{2} u_{k} \\
\vdots \\
\rho f_{n} u_{k}
\end{array}\right], \mathbf{S}=\left[\begin{array}{c}
0 \\
0 \\
0 \\
0 \\
0 \\
s_{1} \\
s_{2} \\
\vdots \\
s_{n}
\end{array}\right], \\
E=e+\frac{1}{2} \rho\left(u_{1}^{2}+u_{2}^{2}+u_{3}^{3}\right)
\end{gathered}
$$

and

$$
p=p\left(\rho, e, \rho f_{1}, \rho f_{2}, \ldots, \rho f_{n}\right) .
$$

These equations include the conservation of total mass, momentum, total energy, and also the physical quantities $\rho f_{i}$, which represent nonequilibrium effects, i.e., either the concentration of chemical species or vibrational energy. The quantities $p, \rho, u_{k}, E$, and $e$ respectively denote the pressure, density, Cartesian velocity components, total and internal energies, whereas the vector $S$ is a set of elements of nonequilibrium source terms. It is 
assumed that an appropriate "frozen" speed of sound $c$ is calculated depending on a gas model, by which Eq.(4) is defined. In this study, a numerical scheme is formulated for the governing equations given by Eqs.(3) and Eq. (4).

\subsection{Two-Temperature model}

In this work, Park's two-temperature model equations[29] are used for calculating nonequilibrium flows. This is among one of the simplest physical models for describing the thermal-chemical nonequilibrium phenomena. It employs an approximation that the translational/rotational energy modes are in equilibrium at the translational temperature, while the vibrational/electronic/electron-translational modes are at the vibrational temperature. The conservative variables of this model include the mixture of vibrationalelectronic energy, $e_{V}$, as well as those of concentration of chemical species:

$$
q=\left(\rho, \rho u_{1}, \rho u_{2}, \rho u_{3}, E, \rho z_{1}, \rho z_{2}, \ldots, \rho z_{n}, e V\right)^{t},
$$

where $z_{3}$ is molar number density per unit mass of species 3 . The chemical reaction rates depend not only on the translational temperature but also on the vibrational temperature, $T_{V}$, which is related to the mixture vibrational-electronic energy $e_{V}$ :

$e_{V}=\sum_{s=m o l} \rho z_{s} e_{v i b}\left(T_{V}\right)+\sum_{s} \rho z_{s} e_{e l e s}\left(T_{V}\right)+\rho z_{e} \frac{3}{2} R T_{V}$.

The vibrational energy $e_{v i b}$, per unit mole is obtained by assuming a harmonic oscillator, and the electronic energy $e_{\text {ele }}$ is calculated by taking accounts of only the first electronic excited state. The conservation equation of $e_{V}$ is given as

$$
\frac{\partial e_{V}}{\partial t}+\frac{\partial e_{V} u_{k}}{\partial x_{k}}=s_{V}
$$

where

$$
\begin{gathered}
s_{V}=\sum_{s=m o l} w_{s} \hat{D}_{s}+\sum_{s=m o l} \rho z_{s} \frac{e_{i i b s}^{*}-e_{v i b s}}{\tau_{s}} \\
-p_{e} \frac{\partial u_{k}}{\partial x_{k}}+2 \rho z_{e} \frac{3}{2} R\left(T-T_{V}\right) \sum_{s} \frac{\nu_{e s}}{M_{s}} \\
+\frac{\partial}{\partial x_{k}}\left[\left(\kappa_{V}+\kappa_{e}\right) \frac{\partial T_{V}}{\partial x_{k}}\right]+\frac{\partial}{\partial x_{k}}\left[\rho \sum_{s} h_{V} D_{s} \frac{\partial z_{s}}{\partial x_{k}}\right] .
\end{gathered}
$$

The first term in $s v$ is the vibrational energy lost or gained due to molecular dissociation or combination. Here, $w$, is chemical production rates of molecular species $s$, and $\hat{D}$, is average vibrational energy per unit mass of molecule $s$, which is created or destroyed at rate $w_{\text {. }}$. The second term is the energy exchange between vibrational and translational( $\mathrm{T}-\mathrm{V})$ modes due to collisions with $e_{v i b}^{*}$, being the vibrational energy in equilibrium with the translational energy. Usually an experimental formula in [30] is used as relaxation time $\tau_{s}$, which, however, has been found to predict a too fast $T$ $\mathrm{V}$ relaration at high temperature. Park introduced two modifications to remedy it. One is the collision limiting of the vibrational relaxation times, and the another is the diffusive T-V relaration[29]. Another formula which takes accounts of high-temperature effects on the $T-V$ relaxation is found in [31]. The third term in $s_{V}$ is the work on electrons done by an electric field induced by the electron pressure gradient. The forth term is the energy exchange due to elastic collisions between electrons and heavy particles where $\nu_{e}$ is collision frequency for electrons and heavy particles. The remainder terms are due to the conduction and diffusion. More detailed description of the two-temperature model is found in [32]. In this study, the 7-species chemical reaction model[33] was used:

(1) $\mathrm{O}_{2}+\mathrm{M} \rightleftharpoons \mathrm{O}+\mathrm{O}+\mathrm{M}, \mathrm{M}=\mathrm{N}, \mathrm{NO}, \mathrm{O}, \mathrm{O}_{2}, \mathrm{~N}_{2}$;

(2) $\mathrm{N}_{2}+\mathrm{M} \rightleftharpoons \mathrm{N}+\mathrm{N}+\mathrm{M}, \mathrm{M}=\mathrm{N}, \mathrm{NO}, \mathrm{O}, \mathrm{O}_{2}, \mathrm{~N}_{2}$;

(3) $\mathrm{NO}+\mathrm{M} \rightleftharpoons \mathrm{N}+\mathrm{O}+\mathrm{M}, \mathrm{M}=\mathrm{N}, \mathrm{NO}, \mathrm{O}, \mathrm{O}_{2}, \mathrm{~N}_{2}$;

(4) $\mathrm{O}+\mathrm{NO} \rightleftharpoons \mathrm{N}+\mathrm{O}_{2}$;

(5) $\mathrm{O}+\mathrm{N}_{2} \rightleftharpoons \mathrm{N}+\mathrm{NO}$;

(6) $\mathrm{O}+\mathrm{N} \rightleftharpoons \mathrm{NO}^{+}+\mathrm{e}^{-}$.

2.3 Numerical Flux in Generalized Coordinates

In practical computation, a numerical flux in generalized coordinates is needed, which we calculate after [7]. Let the vector $\mathbf{n}=\left(n_{1}, n_{2}, n_{3}\right)$ be a normalized cell-interface normal in $\xi$-direction with the vectors $1\left(l_{1}, l_{2}, l_{3}\right)$ and $m\left(m_{1}, m_{2}, m_{3}\right)$ being its recipropal ones: $\mathbf{n} \cdot \mathbf{l}=0 ; \mathbf{n} \cdot \mathbf{m}=0 ; \mathbf{l} \cdot \mathbf{m}=0 ;|\mathbf{n}|=|\mathbf{l}|=|\mathbf{m}|=1$. The normal and tangential velocity components to the cellinterface normal are calculated for each left and right state:

$$
u=n_{i} u_{i}, \quad v=l_{i} u_{i}, \quad w=m_{i} u_{i} .
$$

The numerical flux in the $x$-direction of this local Cartesian coordinates has the form:

$$
F=\left[\begin{array}{c}
\rho u \\
\rho u^{2}+p \\
\rho v u \\
\rho w u \\
\rho H u \\
\rho f_{1} u \\
\rho f_{2} u \\
\vdots \\
\rho f_{n} u
\end{array}\right],
$$

where $H$ is the total enthalpy:

$$
H \equiv \frac{E+p}{\rho} .
$$


Following a specified flux splitting scheme, the numerical flux $\mathrm{F}_{1 / 2}=\left(F_{1}, F_{2}, \ldots, F_{5+n}\right)^{t}$ is calculated in these local Cartesian coordinates. Finally the numerical flux in the $\xi$-direction of the generalized coordinates, $F_{\xi}$, is given as

$$
\mathrm{F}_{\xi}=S\left[\begin{array}{c}
F_{1} \\
n_{1} F_{2}+l_{1} F_{3}+m_{1} F_{4} \\
n_{3} F_{2}+l_{2} F_{3}+m_{2} F_{4} \\
n_{3} F_{2}+l_{3} F_{3}+m_{3} F_{4} \\
F_{5} \\
F_{8} \\
\vdots \\
F_{5+n}
\end{array}\right]
$$

where $S$ is the area of the cell interface. In this procedure the numerical flux in the generalized coordinates is uniquely specified by the definition of the numerical flux in the local Cartesian coordinates, which is discussed in the next section.

\section{NUMERICAL SCHEME}

\subsection{Classification of the FV/DS schemes}

Up to now, the most successful FV/DS schemes have been the Van Leer scheme[25] and the AUSM[26, 27]. These schemes are equipped with favorable properties: conservation of enthalpy for steady flows; small dissipation in the shear layer. As the first step in constructing our scheme, we generalize these schemes, calling them AUSMV-type and AUSMD-type schemes, where "V" and " $\mathrm{D}$ " denote flux-Vector-splitting-biased schemes and flux-Difference-splitting-biased ones, respectively.

It is useful to first review briefly the AUSM scheme. An interesting form for writing the numerical flux is given in [27], as

$\mathbf{F}_{1 / 2}=\frac{1}{2}\left[u_{1 / 2}\left(\Phi_{L}+\Phi_{R}\right)-\left|u_{1 / 2}\right|\left(\Phi_{R}-\Phi_{L}\right)\right]+\mathbf{p}_{1 / 2}$,

where

$$
\begin{gathered}
\Phi=\left(\rho, \rho u, \rho v, \rho w, \rho H, \rho f_{1}, \ldots, \rho f_{n}\right)^{t}, \\
p_{1 / 2}=\left(0, p_{1 / 2}, 0,0,0,0, \ldots, 0\right)^{t} .
\end{gathered}
$$

Here the interface velocity $u_{1 / 2}$ and pressure $p_{1 / 2}$ are defined as

$$
u_{1 / 2}=u_{L}^{+}+u_{R}^{-}
$$

where

$$
u^{ \pm}= \begin{cases} \pm \frac{1}{4 c}(u \pm c)^{2}, & \text { if }|u| \leq c \\ \frac{1}{2}(u \pm|u|), & \text { otherwise }\end{cases}
$$

and

$$
p_{1 / 2}=p_{L}^{+}+p_{R}^{-},
$$

where

$$
p^{ \pm}= \begin{cases}p(u / c \pm 1)^{2}(2 \mp u / c) / 4, & \text { if }|u| \leq c \\ p(u \pm|u|) / 2 u, & \text { otherwise. }\end{cases}
$$

Also Liou and Steffen previously proposed another AUSM scheme, which is based on Mach number splitting[26], where an appropriately defined interface Mach number is used to determine the upwind extrapolation for the convective quantities.

Now, we define the AUSMD-type schemes, which include both of the AUSMs as a member. In defining this type of schemes, we use the interface mass flux, $(\text { ou })_{1 / 2}$, instead of the interface velocity or Mach number as a switching function for the upwind extrapolation. The AUSMD-type schemes are defined by their numerical flux:

$\mathbf{F}_{1 / 2}=\frac{1}{2}\left[(\rho u)_{1 / 2}\left(\Psi_{L}+\Psi_{R}\right)-\left|(\rho u)_{1 / 2}\right|\left(\Psi_{R}-\Psi_{L}\right)\right]+p_{1 / 2}$,

where

$$
\mathbf{\Psi}=\left(1, u, v, w, H, f_{1}, \ldots, f_{n}\right)^{t} .
$$

A specific numerical scheme is uniquely defined by an appropriate interface mass flux $(\rho u)_{1 / 2}$ and interface pressure $p_{1 / 2}$. So far, the interface pressure given by Eq.(15) and Eq.(16) has been commonly used in the existing FV/DS schemes, but the form of the mass flux varies with each numerical scheme. This is because the mass flux is directly connected to the resolution of contact discontinuities, which is a main interest in the FV/DS schemes. For example, the following mass fluxes may be possible for the AUSMD-type scheme, and are actually used in Van Leer's FV/DS and the AUSMs, respectively.

- Van Leer's FV/DS:

$$
(\rho u)_{1 / 2}=u_{L}^{+} \rho_{L}+u_{\bar{R}}^{-} \rho_{R}
$$

- AUSM(velocity-splitting):

$$
(\rho u)_{1 / 2}=\frac{1}{2}\left[u_{1 / 2}\left(\rho_{L}+\rho_{R}\right)-\left|u_{1 / 2}\right|\left(\rho_{R}-\rho_{L}\right)\right],
$$

where

$$
u_{1 / 2}=u_{L}^{+}+u_{R}^{-}
$$

- AUSM(Mach number-splitting):

$$
\begin{aligned}
(\rho u)_{1 / 2}=\frac{1}{2}[ & M_{1 / 2}\left(\rho_{L} c_{L}+\rho_{R} c_{R}\right) \\
& \left.-\left|M_{1 / 2}\right|\left(\rho_{R} c_{R}-\rho_{L} c_{L}\right)\right],
\end{aligned}
$$

where

$$
M_{1 / 2}=u_{L}^{+} / c_{L}+u_{R}^{-} / c_{R}
$$


Here $u^{ \pm}$are defined by Eq.(14). The interface mass flux defined by Eq.(19) or Eq.(20) in conjunction with the interface pressure of Eq.(15) makes the AUSMD-type scheme reduce to the velocity-splitting-based AUSM and the Mach-number-splitting-based AUSM, respectively. It is noted that the mass flux of the Machnumber-splitting-based AUSM vanishes at a stationary discontinuity, making the AUSM a less dissipative scheme among the FV/DS schemes. In the nert section, we will present a new formula for the interface mass flux $(\rho u)_{1 / 2}$ which results in noticeable improvements over the AUSM.

The numerical flux of the Van Leer FV/DS scheme[25] slightly differs from the form of Eq.(17) even if the mass flux of Eq.(18) is used, because that scheme does not use the upwind extrapolation about the term $\left(\rho u^{2}\right)_{1 / 2}$ in the x-momentum flux. Hence, we need to define another class of FV/DS schemes - AUSMV-type schemes:

$$
\left(\rho u^{2}\right)_{\mathrm{AUSMV}}=u_{L}^{+}(\rho u)_{L}+u_{R}^{-}(\rho u)_{R} .
$$

But the AUSMD-type schemes defines

$\left(\rho u^{2}\right)_{\mathrm{AUSMD}}=\frac{1}{2}\left[(\rho u)_{1 / 2}\left(u_{L}+u_{R}\right)-\left|(\rho u)_{1 / 2}\right|\left(u_{R}-u_{L}\right)\right]$.

The mass flux of Eq.(18) and the velocity/pressure splittings of Eqs. $(14,16)$ make the AUSMV-type scheme reduce to the Van Leer FV/DS scheme. We emphasize that a specific AUSMV-type scheme needs the definition of the velocity splitting, $u^{ \pm}$, for the $x$-momentum flux as well as that of the interface mass flux and the pressure. The characteristics of the AUSMD-type and AUSMV-type schemes will be discussed in detail in the following sections.

\subsection{Removal of Numerical Dissipation at Contact Discontinuities}

We discuss first the formula for the interface mass flux because both AUSMD-type and AUSMV-type schemes need it. The main drawback of Van Leer's FV/DS scheme is the numerical viscosity on the contact surface, while that of the AUSM is the numerical overshoot at shock waves. We have found that the overshoot of the AUSM is mainly due to the mass flux differencing. This is probably because the AUSM mass flux of Eq.(19) nor Eq.(20) does not directly take into account of the density behind the shock wave. Hence, in this study we employ a mass flux formula of Eq.(18). In this case, however, the surplus numerical dissipation at the contact discontinuities will be a problem.

First, let's consider a stationary contact discontinuity where $u_{L}=u_{R}=0 ; p_{L}=p_{R}$. The mass flux of Eq.(18) is

$$
(\rho u)_{1 / 2}=\left(\rho_{L} c_{L}-\rho_{R} c_{R}\right) / 4
$$

which does not necessarily vanish, producing the excessive numerical dissipation at the contact discontinuity. The term of $c_{L}$ and $c_{R}$ in Eq.(23) comes from the numerical dissipation which is added other than the simplest velocity splitting: $u^{ \pm}=(u \pm|u|) / 2$. Some additional dissipation, however, is indispensable in order to get a stable scheme. Hence, we modify the velocity splitting so that the numerical dissipation can cancel at the contact discontinuity:

$$
\begin{aligned}
& u_{L}^{+}=\alpha_{L}\left\{\frac{(u+c)^{2}}{4 c}-\frac{u+|u|}{2}\right\}_{L}+\frac{u_{L}+\left|u_{L}\right|}{2} \\
& u_{R}^{-}=\alpha_{R}\left\{-\frac{(u-c)^{2}}{4 c}-\frac{u-|u|}{2}\right\}_{R}+\frac{u_{R}-\left|u_{R}\right|}{2}
\end{aligned}
$$

where

$$
\alpha_{L}=\frac{2 \bar{\alpha}_{L}}{\bar{\alpha}_{L}+\bar{\alpha}_{R}}, \quad \alpha_{R}=\frac{2 \bar{\alpha}_{R}}{\bar{\alpha}_{L}+\bar{\alpha}_{R}},
$$

and

$$
\bar{\alpha}_{L}=\frac{f\left(p_{L}, p_{R}\right)}{(\rho c)_{L}}, \quad \bar{\alpha}_{R}=\frac{f\left(p_{R}, p_{L}\right)}{(\rho c)_{R}}
$$

and " $f$ " is some function of pressure $p_{L}$ and $p_{R}$. Since $p_{L}$ equals to $p_{R}$ at the contact discontinuity, an arbitrary function might be possible as $f$. According to our numerical experiments, we take

$$
f\left(p_{L}, p_{R}\right)=p_{L}, \quad f\left(p_{R}, p_{L}\right)=p_{R}
$$

The velocity splitting, which is a function of $\alpha: 0 \leq \alpha \leq$ 2 , is shown in Fig. 1.

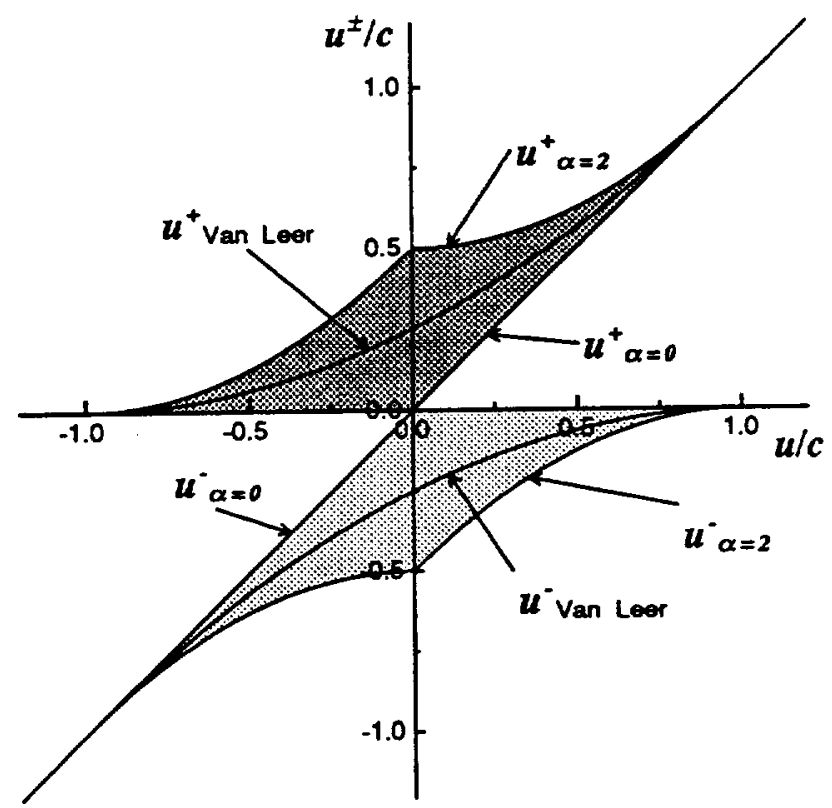

Fig. 1 Velocity splitting. 
This velocity splitting and the interface pressure defined by Eqs. $(15,16)$ make both of the AUSMD/V-type schemes satisfy the condition of no dissipation at the contact discontinuity on every component of the numerical flux vector. It is noted that, if the velocity splitting of Eq. (24) is applied to Van Leer's FVS[21], that scheme is also able to keep the stationary contact discontinuity.

Now, let's consider a moving contact discontinuity: $u_{L}=u_{R}=u_{L R} \neq 0$, where the exact solution of the Riemann problem gives a mass flux:

$$
(\rho u)_{1 / 2}=\frac{1}{2}\left[u_{L R}\left(\rho_{L}+\rho_{R}\right)-\left|u_{L R}\right|\left(\rho_{R}-\rho_{L}\right)\right] .
$$

In this case, the use of a common speed of sound, $c_{m}$, instead of $c_{L}$ and $c_{R}$ in the modified velocity splitting of Eq.(24) enables a mass flux formula of Eq.(18) to reproduce that of Eq.(28). The condition on the momentum flux is also satisfied by the use of the common speed of sound in the pressure splitting of Eq.(16). This simple modification makes all other fluxes reproduce those of the exact Riemann solution for the contact discontinuities. In this study, $\max \left(c_{L}, c_{R}\right)$ is taken as the common speed of sound. We call the specific AUSMD/V-type scheme which has the above interface mass flux and pressure/velocity-splittings as the AUSMD and AUSMV, respectively.

We now summarize the numerical scheme for the AUSMD/V. First, the mass flux is

$$
(\rho u)_{1 / 2}=u_{L}^{+} \rho_{L}+u_{R}^{-} \rho_{R},
$$

where the velocity splittings $u_{L}^{+}, u_{R}^{-}$are no longer the familiar Van Leer splittings, but rather including terms designed to capture stationary/moving contact discontinuities, and are given as

$$
\begin{aligned}
& u_{L}^{+}= \\
& \left\{\begin{array}{cl}
\alpha_{L}\left\{\frac{\left(u_{L}+c_{m}\right)^{2}}{4 c_{m}}-\frac{u_{L}+\left|u_{L}\right|}{2}\right\}+\frac{u_{L}+\left|u_{L}\right|}{2}, & \text { if } \frac{\left|u_{L}\right|}{e_{m}} \leq 1 ; \\
\frac{u_{L}+\left|u_{L}\right|}{2}, & \text { otherwise, }
\end{array}\right.
\end{aligned}
$$$$
u_{R}=
$$$$
\left\{\begin{array}{cl}
\alpha_{R}\left\{-\frac{\left(u_{R}-c_{m}\right)^{2}}{c_{m}}-\frac{u_{R}-\left|u_{R}\right|}{2}\right\}+\frac{u_{R}-\left|u_{R}\right|}{2}, & \text { if } \frac{\left|u_{R}\right|}{c_{m}} \leq 1 ; \\
\frac{u_{R}-\left|u_{R}\right|}{2}, & \text { otherwise, }
\end{array}\right.
$$

where

$$
\alpha_{L}=\frac{2(p / \rho)_{L}}{(p / \rho)_{L}+(p / \rho)_{R}}, \quad \alpha_{R}=\frac{2(p / \rho)_{R}}{(p / \rho)_{L}+(p / \rho)_{R}}
$$

and

$$
c_{m}=\max \left(c_{L}, c_{R}\right)
$$

Secondly, the pressure flux is

$$
p_{1 / 2}=p_{L}^{+}+p_{R}^{-}
$$

where

$p_{L / R}^{ \pm}= \begin{cases}p_{L / R}\left(\frac{u_{L / R}}{c_{m}} \pm 1\right)^{2}\left(2 \mp \frac{u_{L / R}}{c_{m}}\right) / 4, & \text { if } \frac{\left|u_{L / R}\right|}{c_{m}} \leq 1 \\ p_{L / R} \frac{u_{L / R} \pm\left|u_{L / R}\right|}{2 u_{L / R}}, & \text { otherwise. }\end{cases}$

Substitution of Eq.(29) and Eq.(34) into Eq.(17) results in the numerical flux of the AUSMD, in addition, if the term $\rho u^{2}$ in the $x$-momentum fiux is replaced by Eq.(21), we have the scheme AUSMV.

\subsection{AUSMDV: Mixture of AUSMD and AUSMV}

There remains a question about the choice between the AUSMD and AUSMV, i.e., between Eq.(22) and Eq.(21). This will be decided by numerical experiments. First the shock capturing capability is tested by solving a shock-tube problem for ideal gas with specific heat ratio, $\gamma$, of 1.4. The initial condition is $(\rho, p, M)_{L}=$ $(0.1,0.1,15),(\rho, p, M)_{R}=(0.1,0.1,-15)$, which produces strong shock waves propagating in both directions. Figures 2(a), 2(b) and 2(c) show the profiles of temperature, pressure and density after 100 time steps at $C F L=1.0$ with 100 grid points by the AUSM, AUSMD and AUSMV, respectively. The AUSM and AUSMD show noticeable oscillations, although the AUSMD is a little better than the AUSM. On the other hand, the AUSMV gives a fairly good solution in Fig. 2(c), indicating that the AUSMV has a higher shock-capturing capability than the AUSMD.

Another shock-tube problem is a slowly moving contact discontinuity: $(\rho, p, u)_{R}=\left(10.0,1.0,0.3 c_{R}\right)$, $(\rho, p, u)_{L}=\left(0.125,1.0,0.3 c_{R}\right)$ with 30 grid points. Figure $3(\mathrm{a})$ shows the solution after 100 time steps by the AUSMD. Figure $3(b)$ is the solution by the AUSMV. The AUSMV produces serious oscillations, which take place only on the velocity(momentum) field "inside" the left state but not at the contact discontinuity. To clarify the cause of the oscillations, a simplified problem is set up, in which a center grid point is given a small velocity perturbation $(u=0.001 c)$ from a uniform field: $(p, p, u)_{L / R}=(1,1,0)$. Figures $4(a)$ and $4(b)$ are the results after 15 time steps with CFL number of 0.75 and grid number of 31 by the use of the AUSMD and the AUSMV, respectively. For this simple problem, AUSMV produces serious oscillations, although it works well when CFL number is reduced to 0.5. This kind of oscillations, however, are a common problem for the schemes which use a momentum flux formula of Eq.(21): Van Leer's FVS; Hänel's FVS; Hänel's FV/DS; Van Leer's FV/DS. The velocity at the perturbed grid point at the second time step is analytically derived for 
various schemes, and is

$$
u^{n+1} \simeq \begin{cases}(1-\lambda) \varepsilon & \text { :Roe's scheme; } \\ \left(1-\frac{3}{2 \gamma} \lambda\right) \varepsilon & : \text { AUSMD, AUSM; } \\ \left(1-\frac{2 \gamma+3}{2 \gamma} \lambda\right) \varepsilon & : \text { AUSMV } \\ & \text { Van Leer/Hänels' FV (/D)S }\end{cases}
$$

where $\lambda=c \Delta t / \Delta x$ is the CFL number. This analysis indicates that a small CFL number $\leq 2 \gamma /(2 \gamma+3) \simeq 0.48$ should be used for the AUSMV scheme in order to avoid the overshoot in the velocity field. This restriction seems too strong, even though the AUSMV scheme shows a nice shock capturing capability. Hence, we prefer a mixed momentum flux of $(\rho u)_{1 / 2}$ between the AUSMV and AUSMD:

$\left(\rho u^{2}\right)_{1 / 2}=\left(\frac{1}{2}+s\right)\left(\rho u^{2}\right)_{\mathrm{AUSMV}}+\left(\frac{1}{2}-s\right)\left(\rho u^{2}\right)_{\mathrm{AUSMD}}$

where $s$ is a switching function of the pressure gradient:

$$
s=\frac{1}{2} \min \left(1, K \frac{\left|p_{R}-p_{L}\right|}{\min \left(p_{L}, p_{R}\right)}\right)
$$

This a veraging is biased toward to the AUSMV in order to maintain the shock-capturing capability. We call this mixed scheme the AUSMDV. In this study a constant parameter $K=10$ is taken. The AUSMDV is applied to the previous shock-tube problem for colliding flow. The result is shown in Fig. 2(d), which indicates that the AUSMDV has a robust shock-capturing capability. It is noted here that the genuine AUSMV flux is also possible if the CFL restriction is not considered serious. The results for the moving contact discontinuity problem by the use of the AUSMDV and other schemes are found in the Section 4.1.

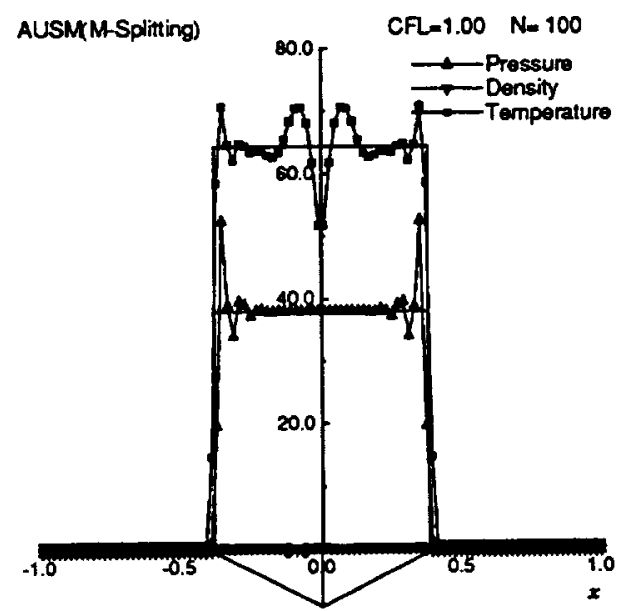

Fig. 2(a) Shock-tube problem - colliding flow by AUSM.

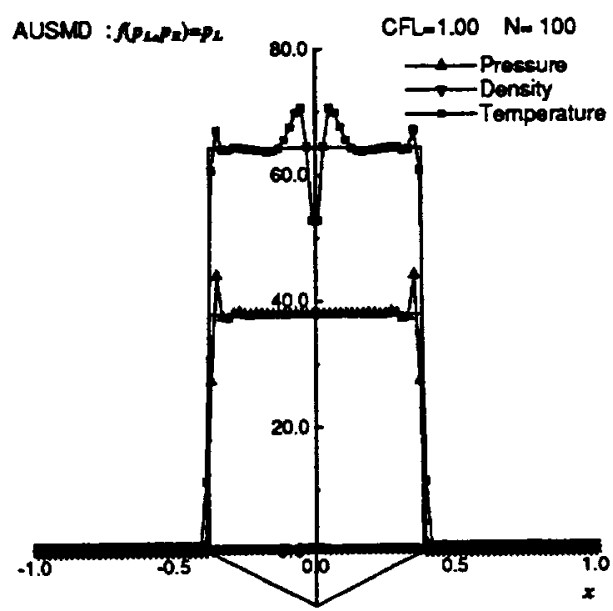

Fig. 2(b) Shock-tube problem - colliding flow by AUSMD.

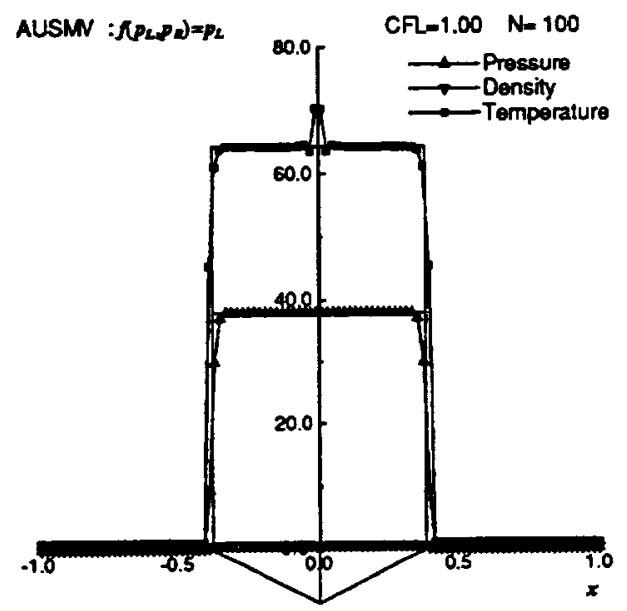

Fig. 2(c) Shock-tube problem - colliding flow by AUSMV.

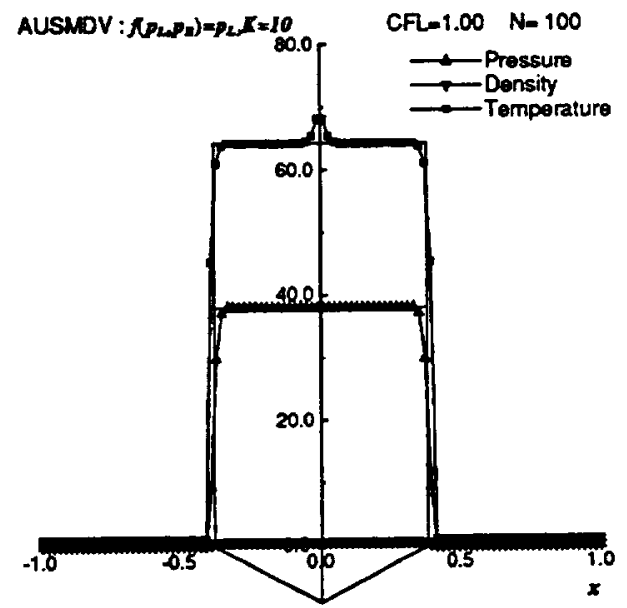

Fig. 2(d) Shock-tube problem - colliding flow by AUSMDV. 


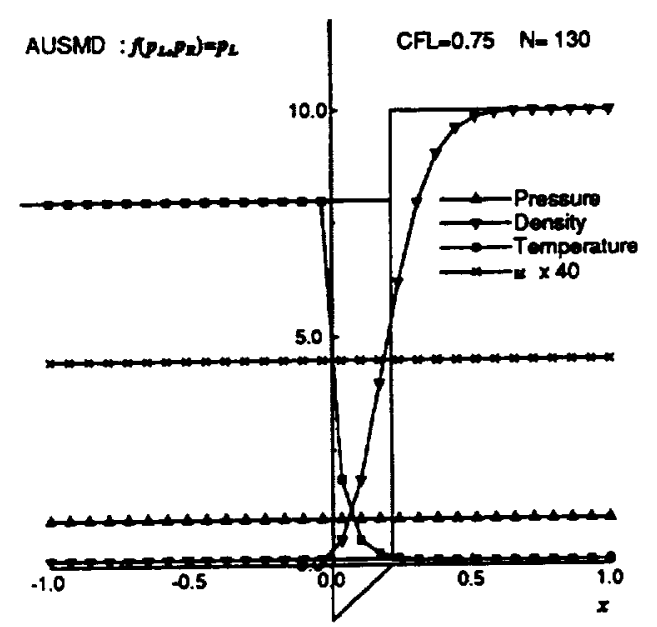

Fig. 3(a) Shock-tube problem - moving contact discontinuity by AUSMD.

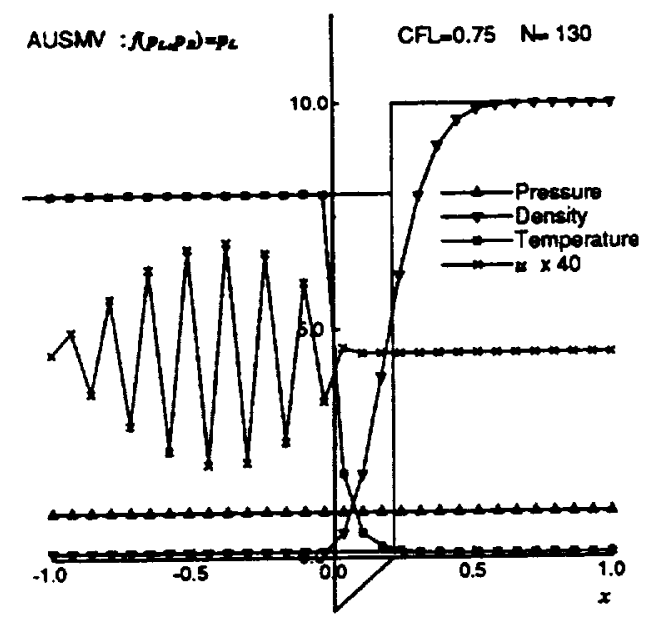

Fig. 3(b) Shock-tube problem - moving contact discontinuity by AUSMV.

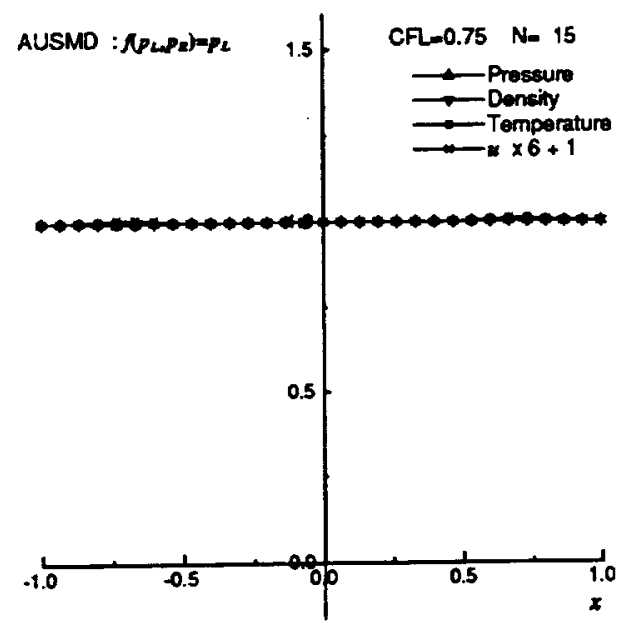

Fig. 4(a) Shock-tube problem - small perturbation on velocity field by AUSMD.



Fig. 4(b) Shock-tube problem - small perturbation on velocity field by AUSMV.

3.4 Shock Fix - a Cure for the Carbuncle Phenomenon

Recently, Quirk[12] has proposed a test problem to investigate the carbuncle phenomenon. The test problem is a shock wave propagating in to a static gas through a duct whose centerline grid is slightly perturbed:

$$
\begin{cases}Y_{j, k m i d}=Y_{k m i d}+\epsilon & \text { for } j \text { even } \\ Y_{j, k m i d}=Y_{k m i d}-\epsilon & \text { for } j \text { odd }\end{cases}
$$

In this study, a unit-spaced $350 \times 19$ grid system is used with the central grid perturbation $\varepsilon$ being $10^{-6}$, and the shock Mach number is 6 .

Figure 5(a) shows the density contonrs solved by Roe's approximate Riemann solver after 400 time steps at CFL number of 1.0, in which the typical carbuncle phenomenon takes place. The same problem was solved by the AUSMDV. That result is shown in Figure 5(b), where the carbuncle phenomenon also emerges although it is less serious than that of the Roe scheme. Other schemes: the exact Riemann solver; the Osher scheme; the HLLE scheme; Van Leer's FVS; Hänel's FVS; the Hänel/Van Leer FV/DS; the AUSM, were tested for this problem. Surprisingly, most of these schemes: the exact Riemann solver; Osher's scheme; the Hānel/Van Leer FV/DS, suffer from the carbuncle phenomenon, although degree of instability may vary. It is noticeable that the AUSM is free from the carbuncle phenomenon even though it is a less dissipative scheme. However, the carbuncle phenomenon seems to be a rather common problem for usual shock-capturing schemes. 

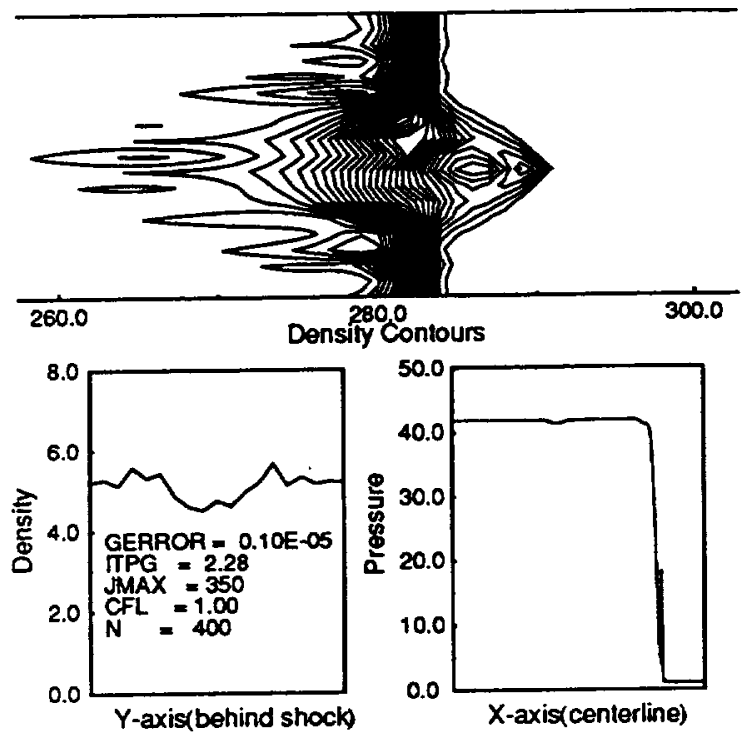

Fig. 5(a) Shock wave propagating through a duct by Roe scheme.
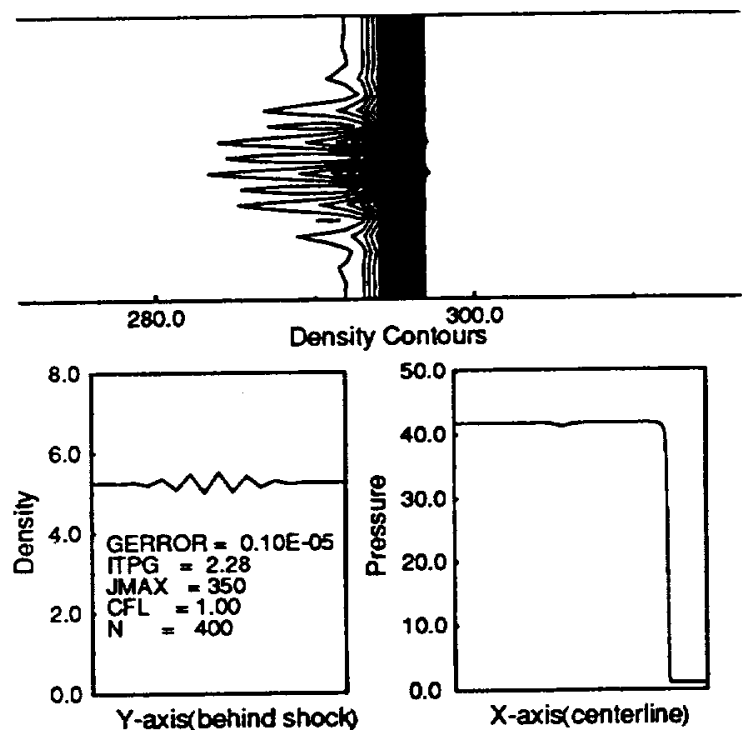

Fig. 5(b) Shock wave propagating through a duct by AUS$\mathrm{MDV}$.

We suspect that the carbuncle phenomenon comes from the recognition of a multi-dimensional shock wave by a one-dimensional numerical scheme. When the shock wave is captured by a shock capturing scheme, an intermediate point(s), which is unphysical, is needed to express a shock position numerically. In 1-D case, if the scheme is well designed, this is not a problem. But when that scheme is applied to multi-dimensional calculation, the intermediate point exchanges information with the neighbors which are also intermediate shock points. It is probable that the exchange of information between these unphysical data causes a numerical instability. In that case, a mechanism to stabilize the shock or some form of artificial viscosity is required. We call this artificial procedure "Shock-Fix." Here, the following shock-fix is proposed.

1. Find out compressible sonic points across the cell interface before calculating numerical flux. This sonic point takes place when

$$
\left\{\begin{array}{rll}
u_{L}-c_{L}>0 & \text { and } & u_{R}-c_{R}<0 \\
\text { or } & u_{L}+c_{L}>0 \text { and } & u_{R}+c_{R}<0
\end{array}\right.
$$

If this is detected between grid point $j$ and $j+1$, set flag $S_{\xi, j}=1$ and $S_{\xi, j+1}=1$ in $\xi$-direction. The same procedure is done in $\eta / \zeta$-direction, which is easy to vectorize.

2. In calculating a numerical flux in $\xi$-direction between grid points $j$ and $j+1, S_{\eta}$ and $S_{\zeta}$ but $S_{\xi}$ are used to sense the shock position, to which some dissipative scheme is applied. That is

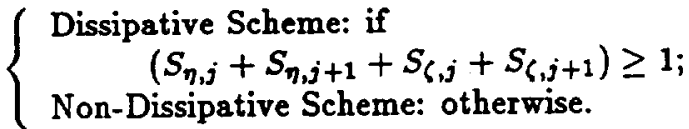

The numerical fluxes in other directions are calculated in a similar way.

This method unlike [12] does not use the pressure gradient in sensing shock waves. The pressure gradient does not always provide a sufficient information for the recognition of shock wave, since in that case some empirical parameter to distinguish shock and compressive wave would be needed. It is noted that the alone shock fix procedure is easily applicable to unstructured grid approach. This shock-fix needs a dissipative scheme to stabilize the shock wave. For the AUSMDV, we employ Hänel's FVS scheme, because it is dissipative enough and conserves the total enthalpy for steady flows. The numerical flux of Hänel's FVS is

$$
\mathbf{F}_{1 / 2}=\rho_{L} u_{L}^{+} \Psi_{L}+\rho_{R} u_{R}^{-} \Psi_{R}+\mathbf{p}_{1 / 2}
$$

where the velocity splitting and the interface pressure are defined in Eq.(14) and Eqs. $(15,16)$, respectively.

Figure $5(\mathrm{c})$ shows the solution by the AUSMDV with the proposed shock-fix, where the carbuncle phenomenon is removed now. A similar shock-fix is possible for Roe's approximate Riemann solver, for which the HLLE scheme is considered to be a dissipative partner scheme. The solution of the Roe scheme with this shock-fix is shown in Figure 5(d), where the instability is totally eliminated. This indicates the validity of the proposed cure of the carbuncle phenomenon, and seems to support our suspicion about its origin. 


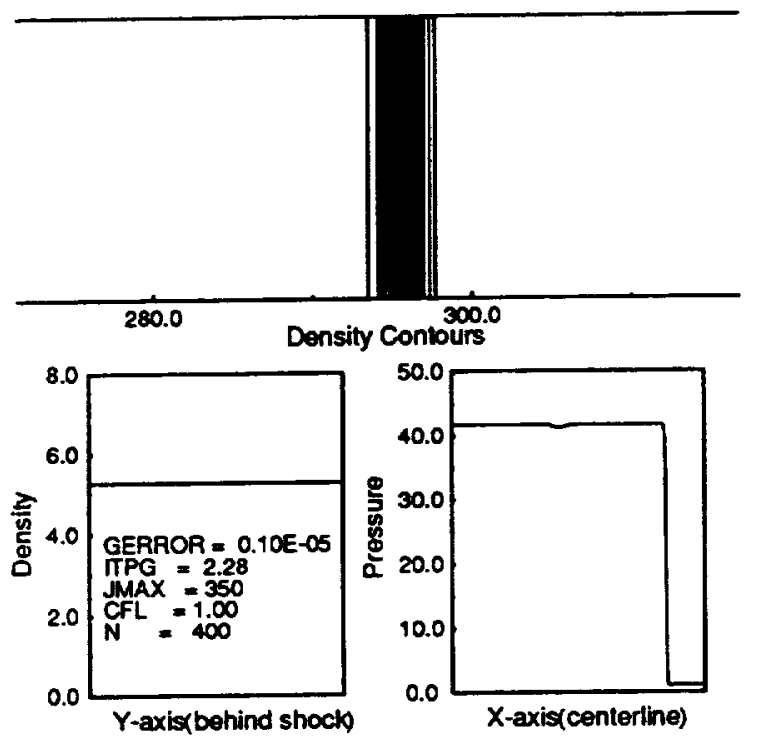

Fig. 5(c) Shock wave propagating through a duct by AUSMDV with shock-fix.
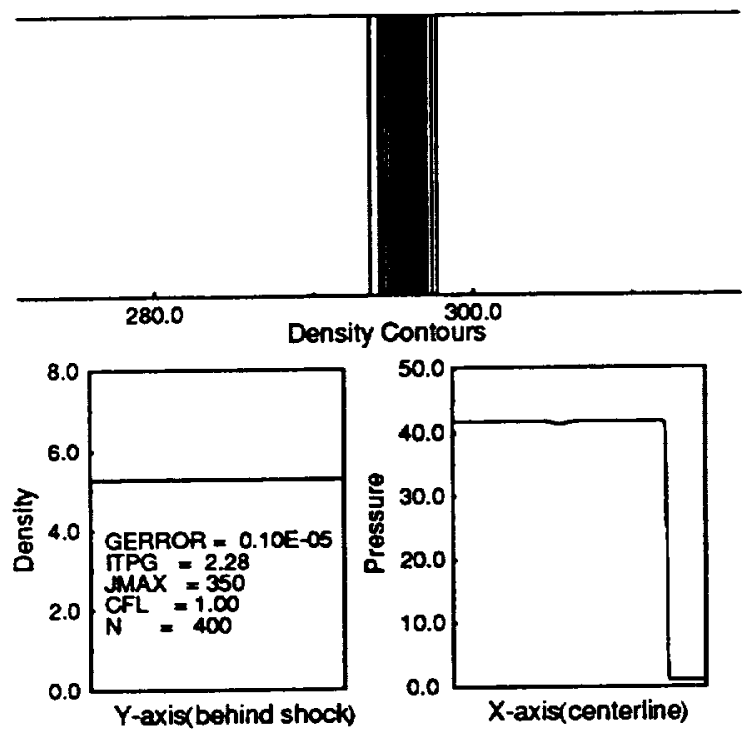

Fig. 5(d) Shock wave propagating through a duct by Roe scheme with shock-fix.

\subsection{Entropy Fix}

It is well known that the Roe scheme without entropy-fix gives an expansion shock. But also the Godunov scheme(exact Riemann solver) produces glitches at sonic points[34, 35]. To investigate this problem, a shock-tube problem with an initial condition of the inverse shock of Mach number 15 was solved. Figures $6(a)$ and $6(\mathrm{~b})$ show the density profile on a mesh of 100 points after 50 time steps at CFL number of 1.0 , using the Roe scheme and the Godunov scheme, respectively. The Roe scheme keeps this unphysical discontinuity, while the Godunov scheme spreads it out but still has a slight glitch at the sonic point. The AUSMDV scheme also produces a similar glitch in Fig. 6(c).

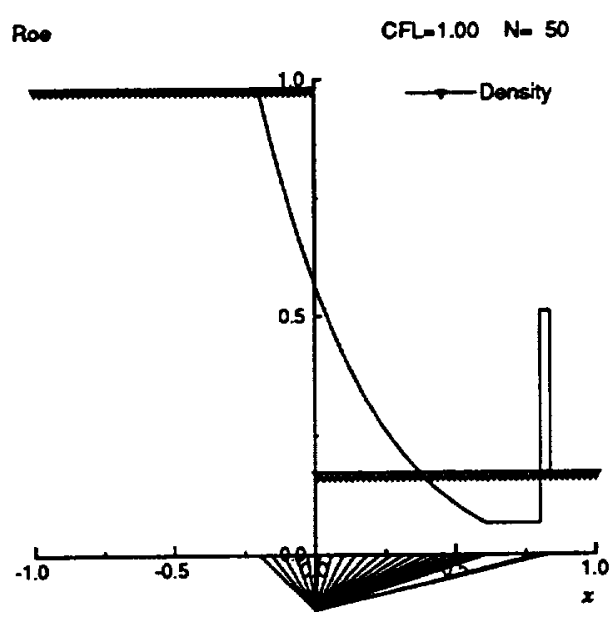

Fig. 6(a) Shock-tube problem - inverse shock wave by Roe scheme.

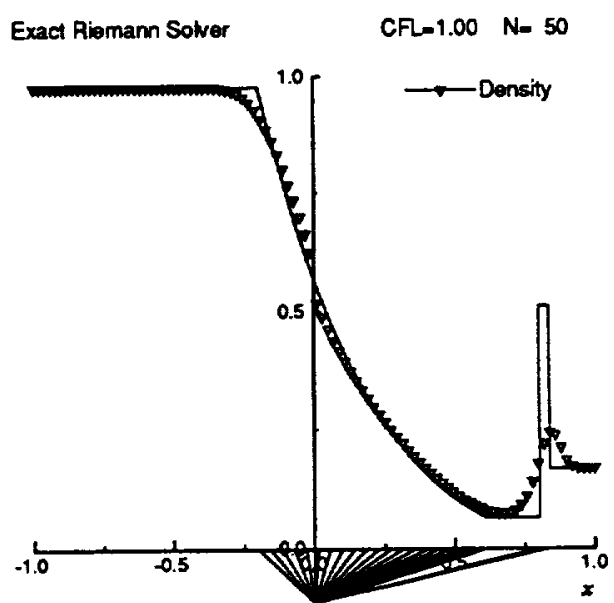

Fig. 6(b) Shock-tube problem - inverse shock wave by Godunor scheme.

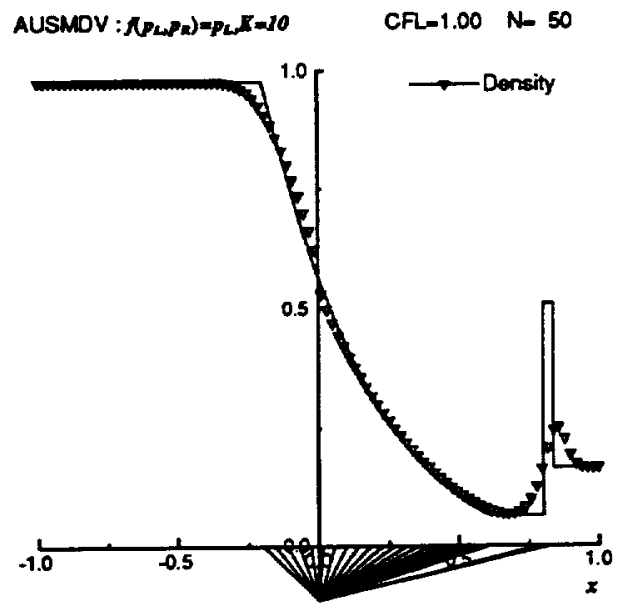

Fig. 6(c) Shock-tube problem - inverse shock wave by AUSMDV.

Usually the numerical flux near the sonic point needs some modification to remove this glitch. In this study, the following modification is proposed. There are two 
cases where an expansive sonic point is found across the cell interface:

$$
\begin{cases}\text { Case A: } & u_{L}-c_{L}<0 \text { and } u_{R}-c_{R}>0 \\ \text { Case B: } & u_{L}+c_{L}<0 \text { and } u_{R}+c_{R}>0\end{cases}
$$

Then we introduce a numerical dissipation only when a single expansion wave is detected, modifying the flux $F_{1 / 2}$ to $F_{1 / 2, E-F_{i x}}$ :

if $A$ and $B)$ then no modification

elseif ( A ) then

$$
\mathrm{F}_{1 / 2, B-F i x}=\mathbf{F}_{1 / 2}-C \Delta(u-c) \Delta(\rho \Psi)
$$

elseif ( B ) then

else

$$
\mathbf{F}_{1 / 2, E-F i x}=\mathbf{F}_{1 / 2}-C \Delta(u+c) \Delta(\rho \mathbf{\Psi})
$$

endif

$$
\text { no modification }
$$

where $\Delta() \equiv()_{R}-()_{L}$, and a constant parameter $C$ is taken 0.125 . This entropy-fix keeps the enthalpyconservation property of the AUSMDV. Figure 6(d) is the shock-tube solution by the AUSMDV with this entropy-fix, where the glitch is fairly removed.

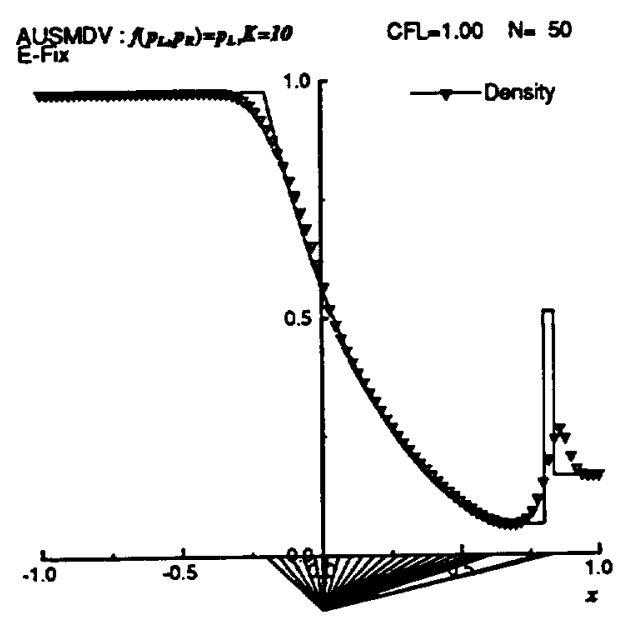

Fig. 6(d) Shock-tube problem - inverse shock wave by AUSMDV with entropy fix.

Another numerical example is a strong shock diffracting around a corner[12]. Figure $7(a)$ and $7(b)$ show the density contours by the Godunov scheme and the AUSMDV without entropy-fix, respectively. These solutions are similar, and give rise to a nearly discontinuous expansion fan. Figure $7(c)$ is the solution by the AUSMDV with the entropy-fix, where the discontinuity is removed fairly well. The inner shock wave of the AUSMDV looks weaker than that of the Godunov scheme, but its strength seems to be related to the glitch of the expansion fan, for the Roe scheme with the entropyfix[34] gives almost the same solution as the entropyfixed AUSMDV in Fig.7(c). It is noted that the Roe scheme without the entropy-fix and the Osher scheme failed in this problem.

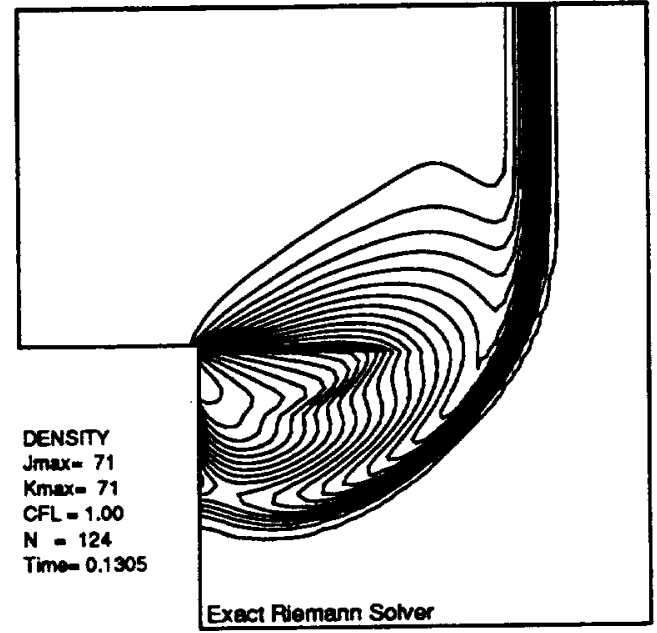

Fig. 7 (a) A shock wave diffracting around a corner by Godunov scheme.



Fig. 7(b) A shock wave diffracting around a corner by AUSMDV without entropy-fix.



Fig. 7(c) A shock wave diffracting around a corner by AUSMDV with entropy-fix. 


\section{NUMERICAL RESULTS AND DISCUS- SIONS}

Various numerical experiments, including a thermochemical nonequilibrium flow problem, are performed in order to verify the soundness of the AUSMDV with the entropy/shock-fix.

\subsection{One-Dimensional Shock-Tube Problems}

Probably, a theoretical drawback of the AUSMDV lies in its shock-capturing capability(SCC). The AUSMDV is equipped with the properties: high-resolution for contact discontinuities; conservation of enthalpy for steady flows. They are obtained at the sacrifice of the theoretical basis of the SCC of Van Leer's FVS, where the flux splittings are wisely designed to have degeneracy in subsonic region so as to build a stationary shock structure with no more than two interior zones. Hence the SCC of the AUSMDV should be assured by numerical experiments. For this purpose, several shock-tube problems were solved.

Figure $8(\mathrm{a})$ is a solution by the AUSMDV scheme for a steady shock at Mach number of 25 , where a primitivevariable-averaged intermediate state is inserted at the $x=0$ point as an initial condition. This figure indicates the AUSMD holds almost the same SCC as that of Van Leer's FVS, producing a monotonic and crisp shock profile. Figure $8(b)$ is the solution of the AUSM based on Mach number splitting[26], showing an overshoot. It is interesting, however, to see that the temperature has almost no overshoot, suggesting that the overshoot of the AUSM is mainly due to density field(mass flux). Also it is noted that this overshoot depends on the initial condition. When the intermediate point was not inserted, the AUSM gave a smoother solution.

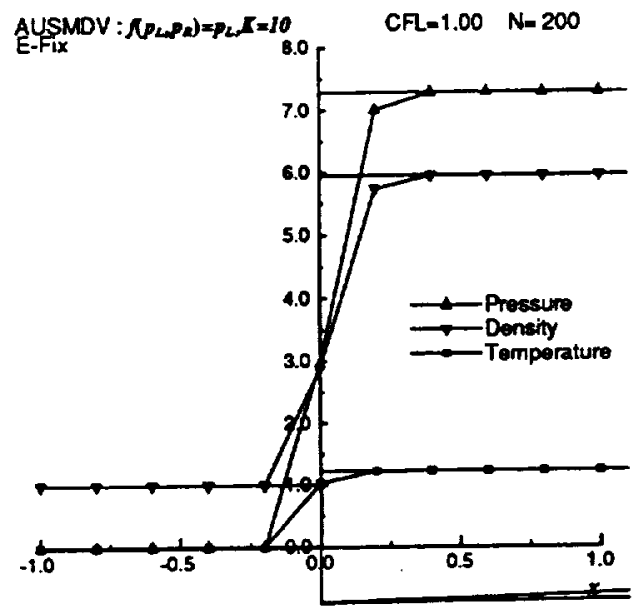

Fig. 8(a) Shock-tube problem - stationary shock by AUSMDV.



Fig. 8(b) Shock-tube problem - stationary shock by AUSM.

Another concern about SCC is a noise made by a slowly-moving shock wave. Roberts[18] proposed a test problem, in which a shock traverses at Mach number of 3 in a computational domain of 100 grid points, passing a single cell in approximately 50 time steps at a CFL number of 0.95 . Figures $9(\mathrm{a}-\mathrm{f})$ show the strength of linear and nonlinear waves after 2000 time steps by the AUSMDV, the AUSM, Van Leer's FVS, Roe's FDS, the HLLE and Osher's scheme, respectively. Here, the Roe linearization was used to compute the wave strength. The Roe scheme and the HLLE, which belong to the Godunov-type scheme, produce a significant error behind the shock wave. Other schemes give much better solutions, although the AUSM has a small overshoot on $u-c$ wave. It may be noticed that the AUSMDV, AUSM and Van Leer's FVS have undershoot on $u$ wave, which was also a common problem for the Osher scheme during the computation. But this undershoot is confined near the shock wave, and is not considered serious. The Godunov-type schemes, on the other hand, leave strong perturbation with long wave-length behind the shock wave.

The next problem is a moving contact discontinuity, of which condition is the same as in Section 3.3. Figures $10(a-f)$ is the results by the AUSMDV, the AUSM, the Godunov scheme, the HLLE, Van Leer's FVS, Van Leer's FV/DS, respectively. The solution of the AUSMDV is identical to that of the exact Riemann solver. Also Roe's scheme and Osher's scheme give the same result. The solution of the AUSM is fairly good although slight oscillations are found in the pressure and velocity fields. The other schemes are unacceptable. The HLLE is too dissipative, and the other FV/DS schemes diverged before 100 time steps, so Figures $10(\mathrm{e})$ and $10(f)$ show the solutions at only 15-th time step. 


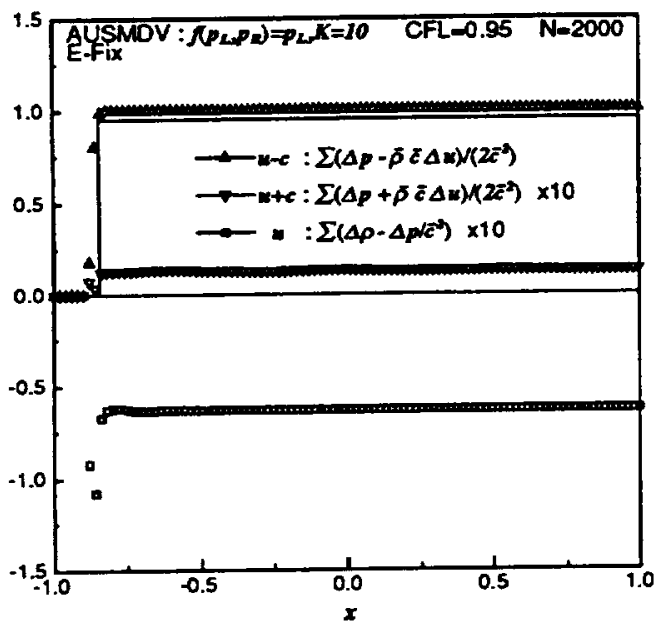

Fig. 9(a) Shock-tube problem - slowly moving shock wave by AUSMDV.



Fig. 9(b) Shock-tube problem - slowly moving shock wave by AUSM.

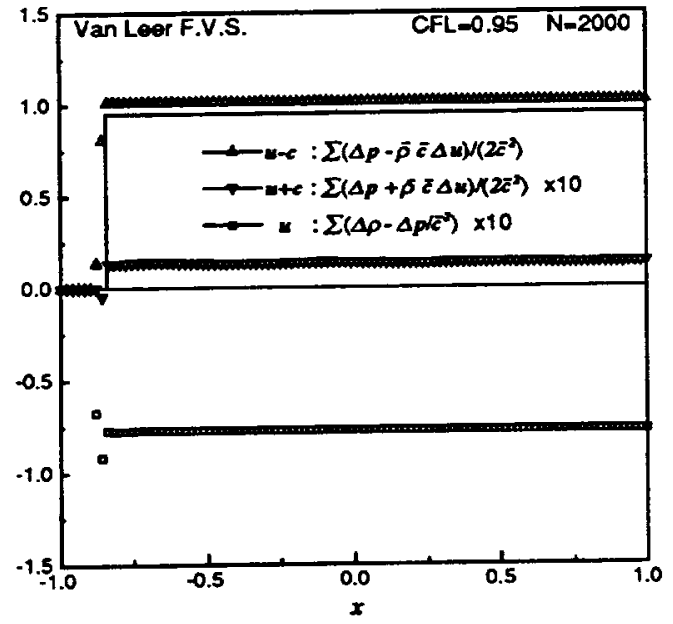

Fig. 9(c) Shock-tube problem - slowly moving shock wave by Van Leer's FVS.

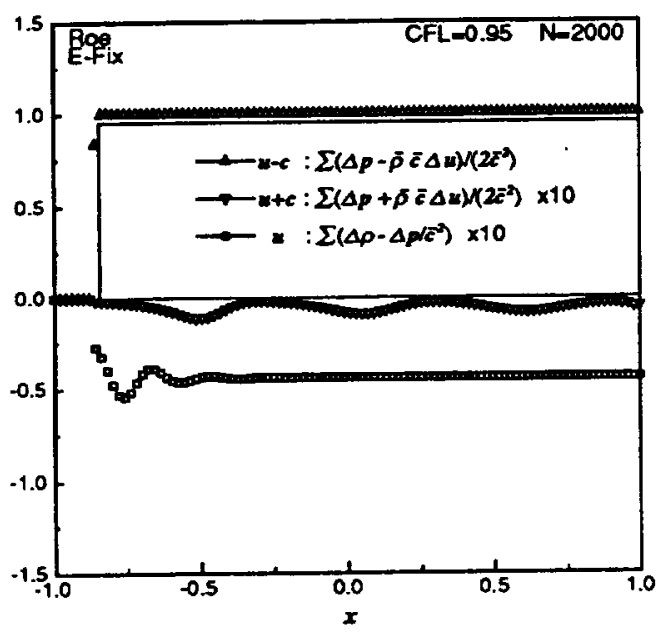

Fig. 9(d) Shock-tube problem - slowly moving shock wave by Roe scheme.

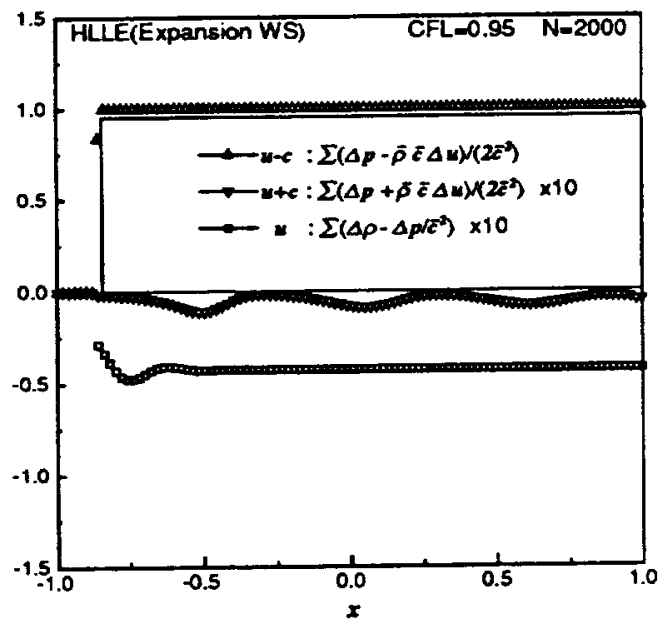

Fig. 9(e) Shock-tube problem - slowly moving shock wave by HLLE scheme.

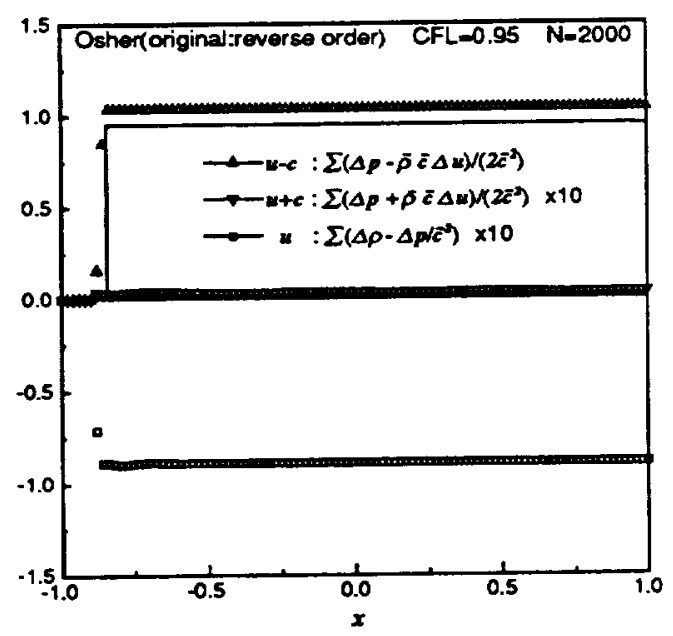

Fig. 9(f) Shock-tube problem - slowly moving shock wave by Osher scheme. 


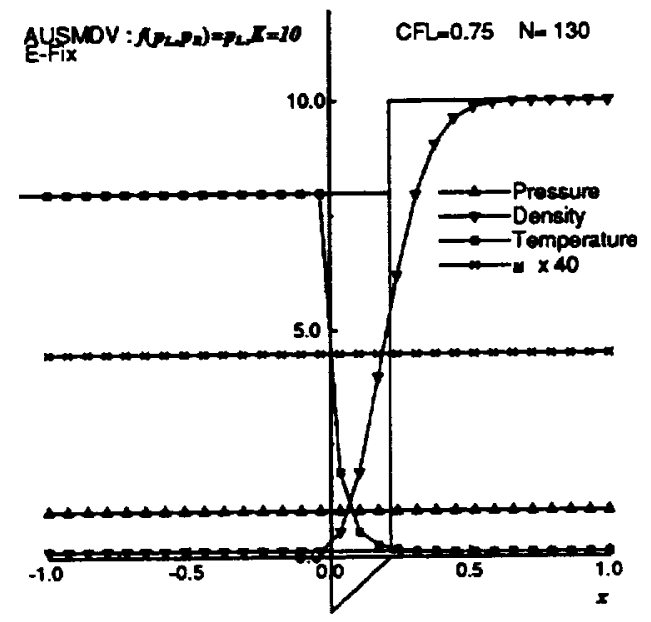

Fig. 10(a) Shock-tube problem - moving contact discontinuity by AUSMDV.

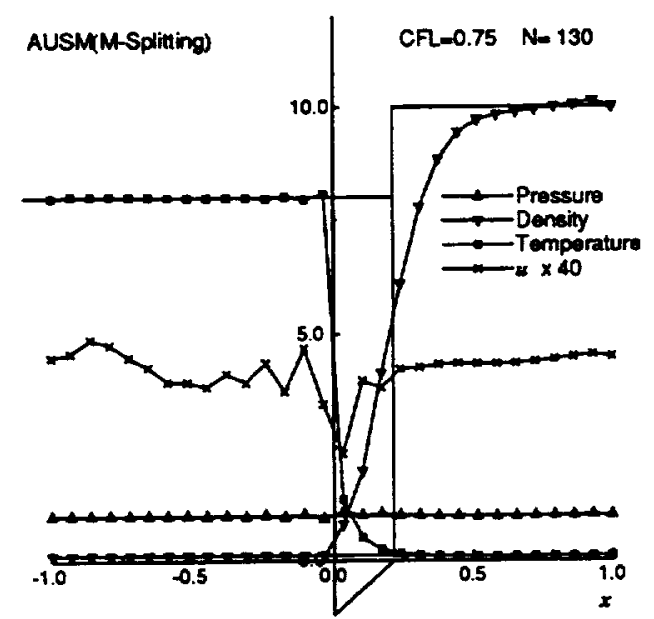

Fig. 10(b) Shock-tube problem - moving contact discontinuity by AUSM.

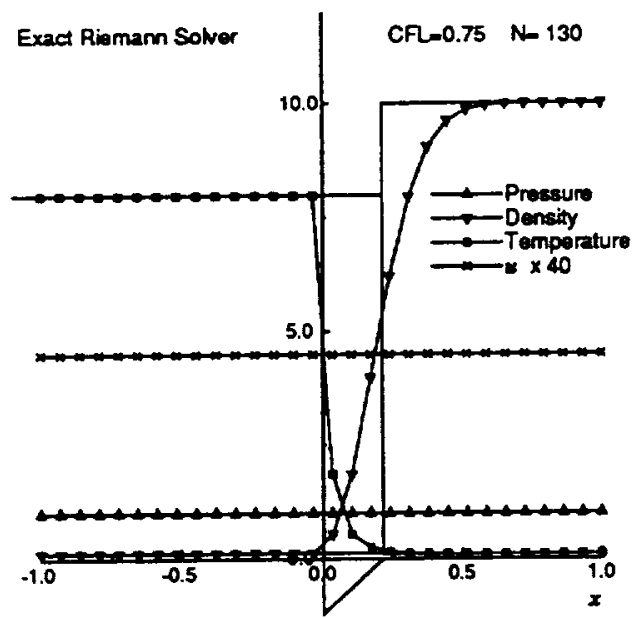

Fig. 10(c) Shock-tube problem - moving contact discontinuity by Godunov scheme.

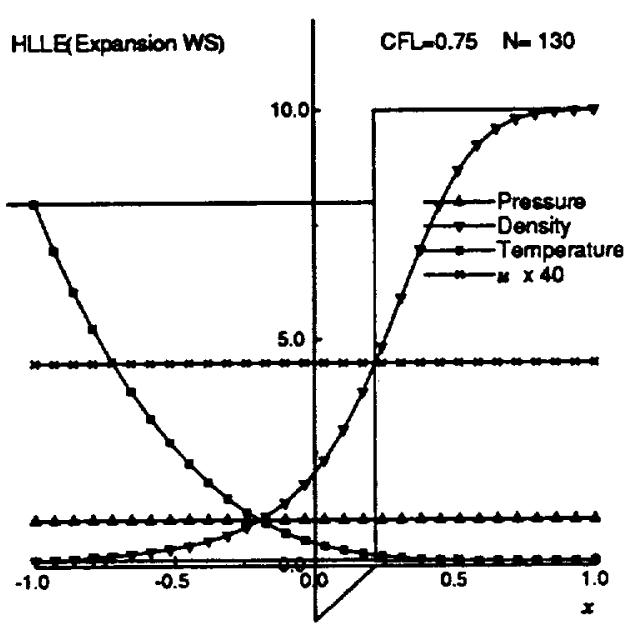

Fig. 10(d) Shock-tube problem - moving contact discontinuity by HLLE scheme.

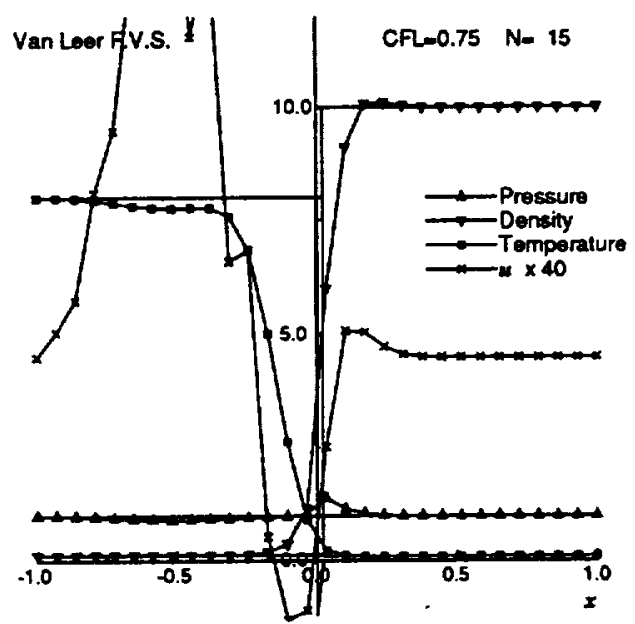

Fig. 10(e) Shock-tube problem - moving contact discontinuity by Van Leer's FVS.



Fig. 10(f) Shock-tube problem - moving contact discontinuity by Van Leer's FV/DS. 
Figures 11(a-c) show the solntion of Sod's problem: $(\rho, p, u)_{L}=(1,1,0) ;(\rho, p, u)_{R}=(1 / 8,1 / 10,0)$ by the AUSMDV, the AUSM and the Roe scheme, respectively. The AUSMDV gives almost the same result as the Roe scheme, while the AUSM shows slight overshoot and undershoot at the shock and the tail of expansion fan although it gives more accurate resolution of the fan.

Finally, the robustness of the AUSMDV is verified by the use of a shock-tube problem with strong expansion fans. The initial condition is $(\rho, p, u)_{L}=$ $\left(1,2,-2.5 c_{L}\right) ;(\rho, p, u)_{R}=\left(1,0.5,2.5 c_{L}\right)$. Figures 12 (ac) are the solutions by the AUSMDV, the Godunov scheme and the HLLE, respectively. The AUSMDV gives a nice solution with no glitch. The Godunov scheme gives also a satisfactory solution, which was very similar to that by the AUSM, Van Leer's FVS and Van Leer's FV/DS, but there is a glitch at the sonic point. The HLLE scheme has no glitch, but its excessive dissipation gives a bump profile in the temperature. It is noted that Roe's scheme and Osher's scheme failed in this problem.

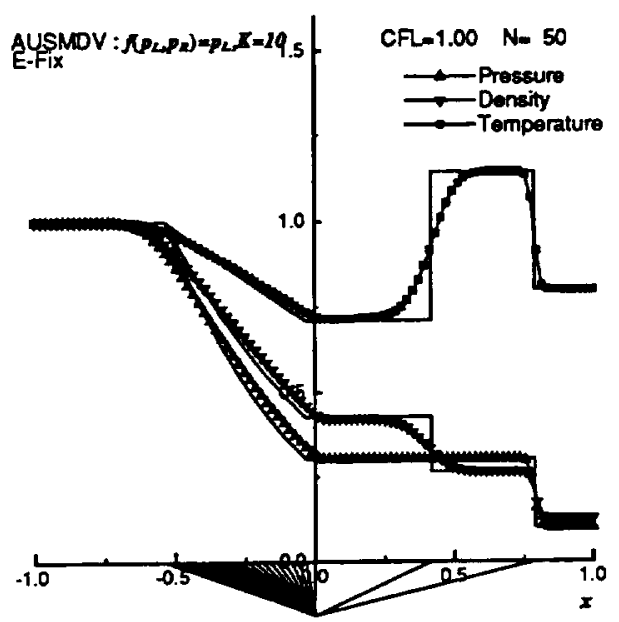

Fig. 11(a) Sod's shock-tube problem by AUSMDV.



Fig. 11(b) Sod's shock-tube problem by AUSM.

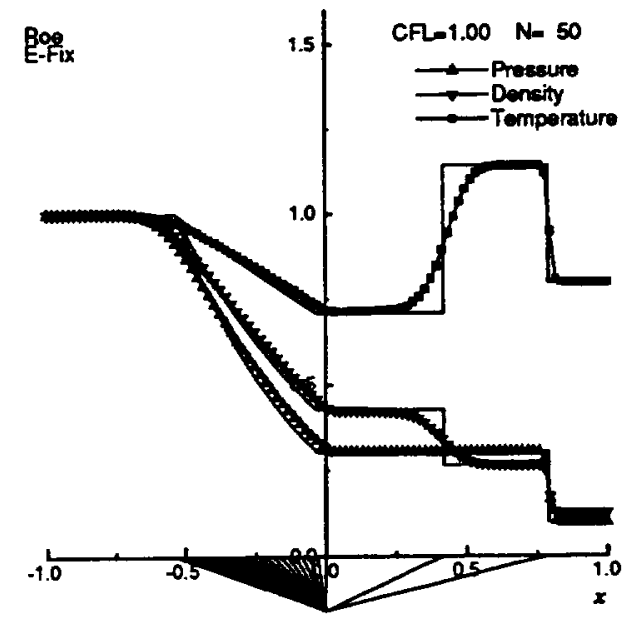

Fig. 11(c) Sod's shock-tube problem by Roe scheme.

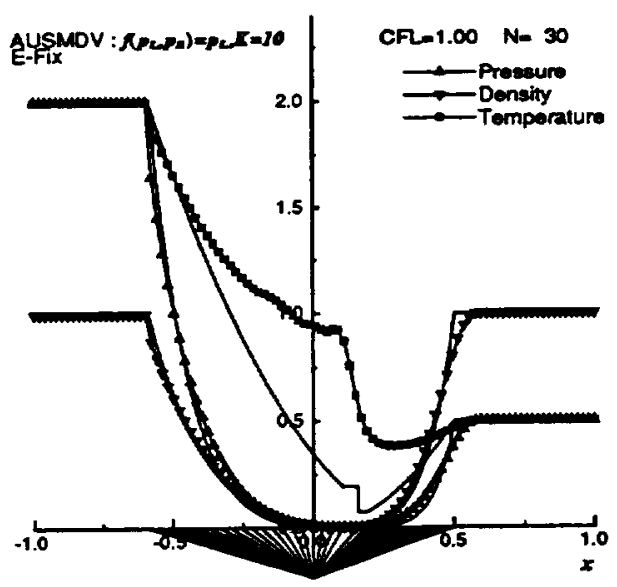

Fig. 12(a) Shock-tube problem - strong expansion by AUSMDV.

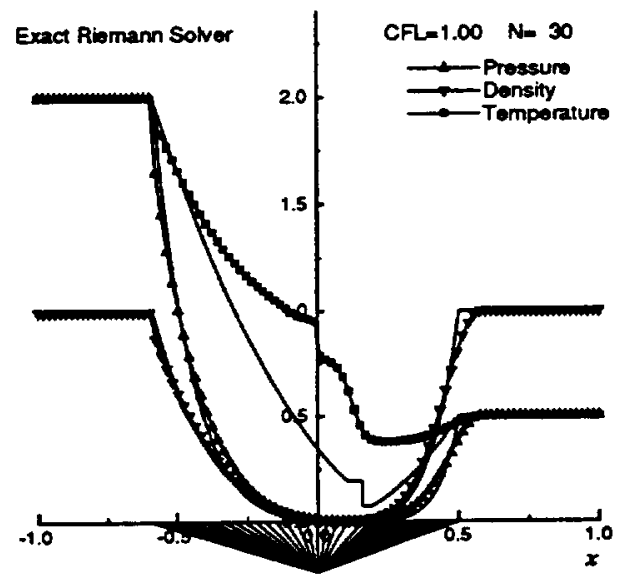

Fig. 12(b) Shock-tube problem - strong expansion by Godunov scheme. 


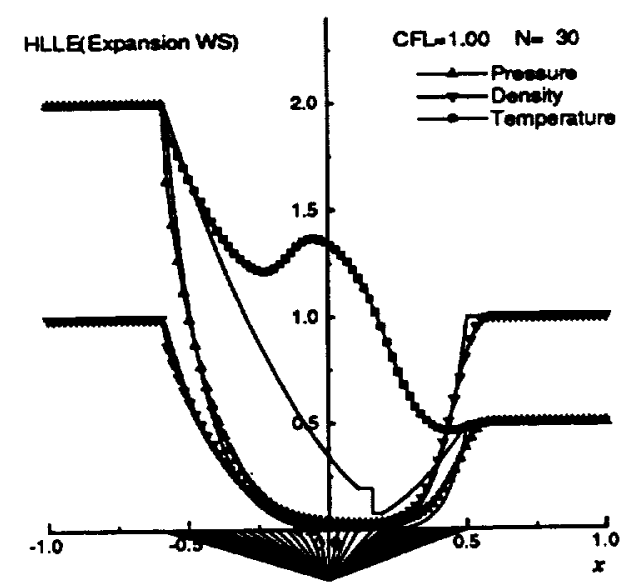

Fig. 12(c) Shock-tube problem - strong expansion by HLLE scheme.

\subsection{Two-Dimensional Shear Flow}

The second problem is a two-dimensional shear flow through a constant-area duct using the Euler equations. Two supersonic inlet flows divided at the central grid are assumed with the left boundary condition: $(\rho, p, M)_{\text {upper }}=(1,1,2) ;(\rho, p, M)_{\text {lower }}=(10,1,1.1)$. Here a very coarse grid system of $10 \times 10$ was used. Figures $13(a)$ shows the density contours and other quantities solved by the AUSMDV, which maintains a strong shear layer. The Godunov scheme, Roe scheme, Osher scheme and the AUSM also gave the same solution. Figures 13(b-d) show the results by Van Leer's FVS, Hänel's FV/DS, Van Leer's FV/DS, respectively. These schemes can not keep the shear layer although Van Leer's FV/DS shows a noticeable improvement over the Hänel's FV/DS. It is noted that the HLLE scheme was as dissipative as the Van Leer FVS.

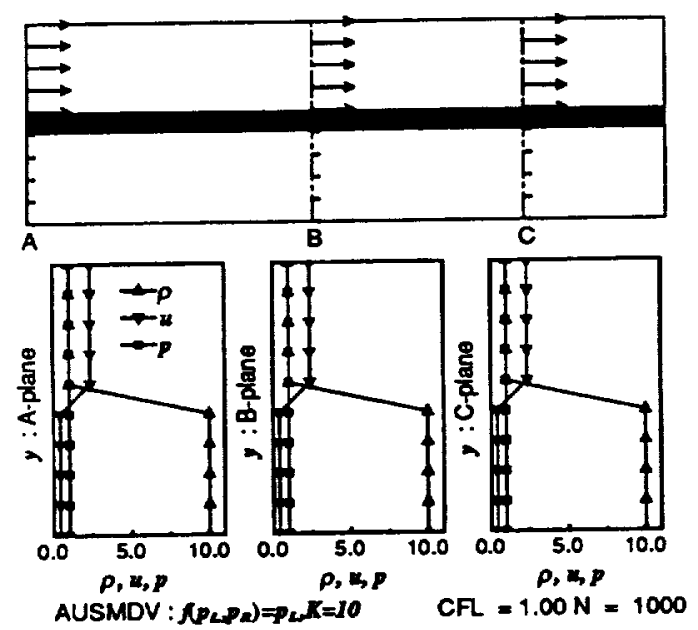

Fig. 13(a) Two-dimensional shear flow problem by AUSMDV.

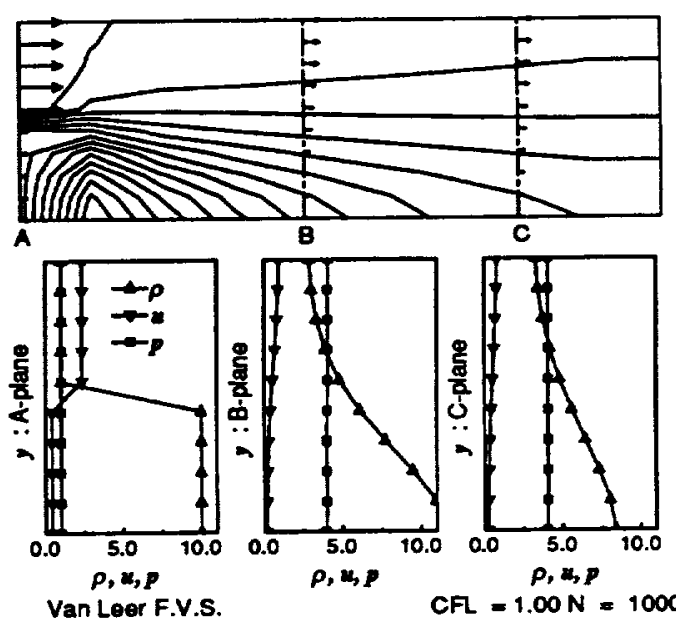

Fig. 13(b) Two-dimensional shear flow problem by Van Leer's FVS.

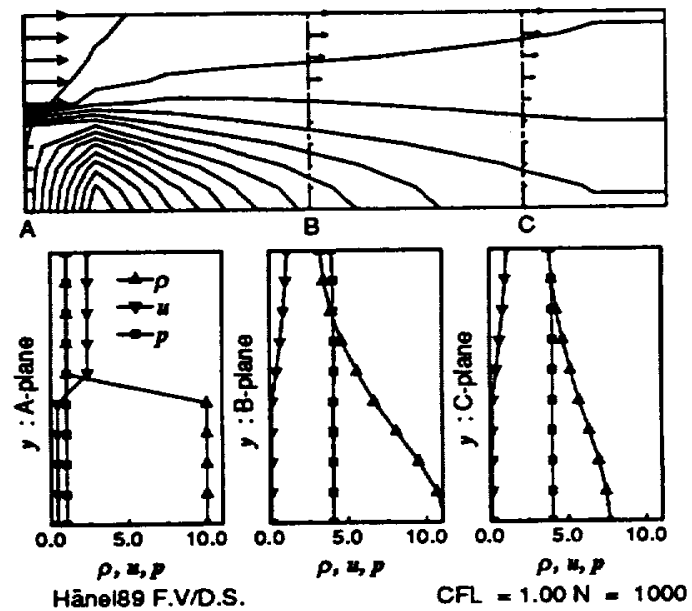

Fig. 13(c) Two-dimensional shear flow problem by Hänel's FV/DS.

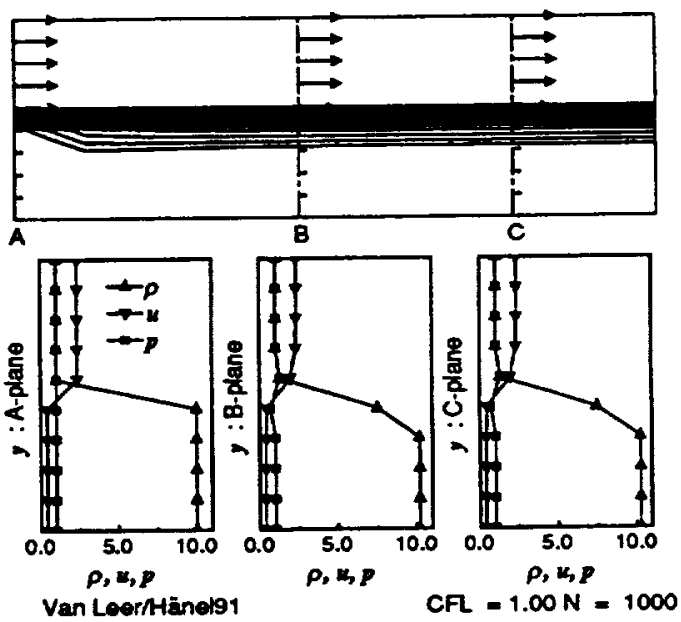

Fig. 13(d) Two-dimensional shear flow problem by Van Leer's FV/DS. 
4.3 One-Dimensional Self-Similar Conical Flow Problem

The third problem is also a standard problem: 1D self-similar conical flow over a 10-degree half cone at hypersonic speed. This problem is used to check the accuracy of numerical schemes on the shock and boundary layer[36]. The flow conditions are $M_{\infty}=7.95 ; R e_{\infty}=$ $4.2 \times 10^{5}$. Figures $14(\mathrm{a})$ and $14(\mathrm{~b})$ show the profiles of pressure, temperature and tangential velocity solved by the AUSMDV and the Roe scheme, respectively. Since $\operatorname{Pr}=1.0$, the exact solution of adiabatic wall temperature is available and indicated by an arrow. The Roe scheme and the AUSMDV give similar results although the latter has a slightly steep curvature in the pressure field at the edge of the boundary layer. For this problem, Van Leer's FVS is known to thicken the boundary layer, as is shown in Fig. 14(c). Also Van Leer's FV/DS gives a glitch[25] in Fig. 14(d).



Fig. 14(a) Conical flow problem by AUSMDV.

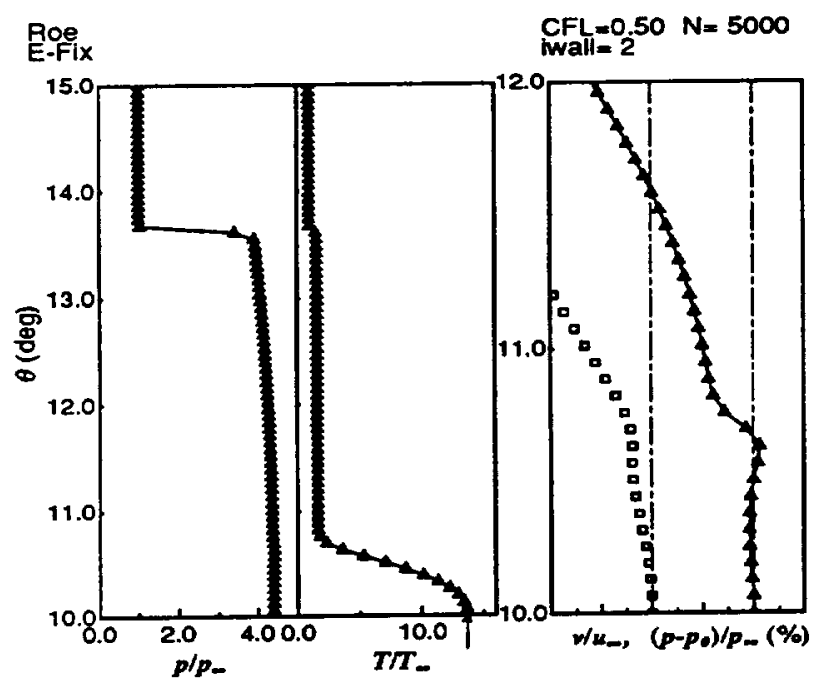

Fig. 14(b) Conical flow problem by Roe scheme.

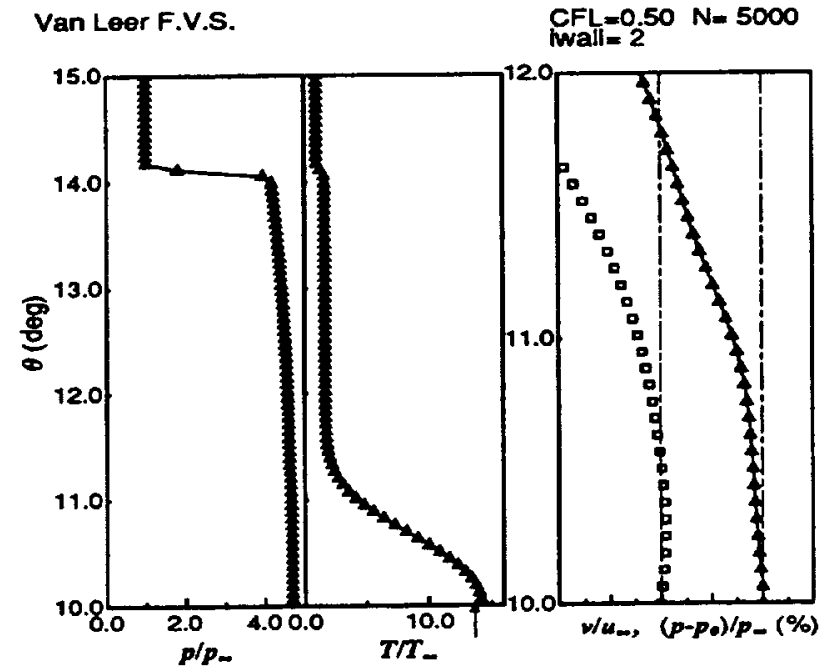

Fig. 14(c) Conical flow problem by Van Leer's FVS.

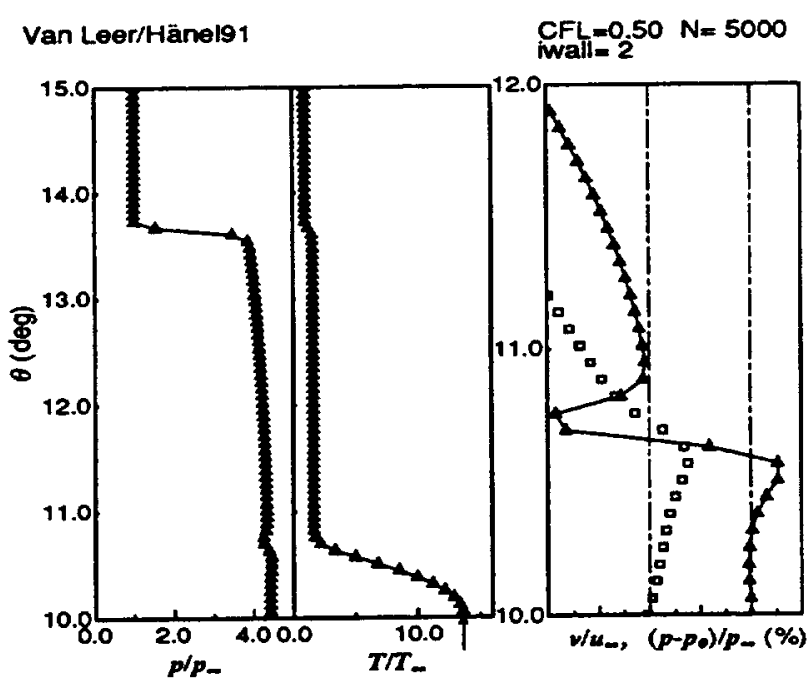

Fig. 14(d) Conical flow problem by Van Leer's FV/DS.

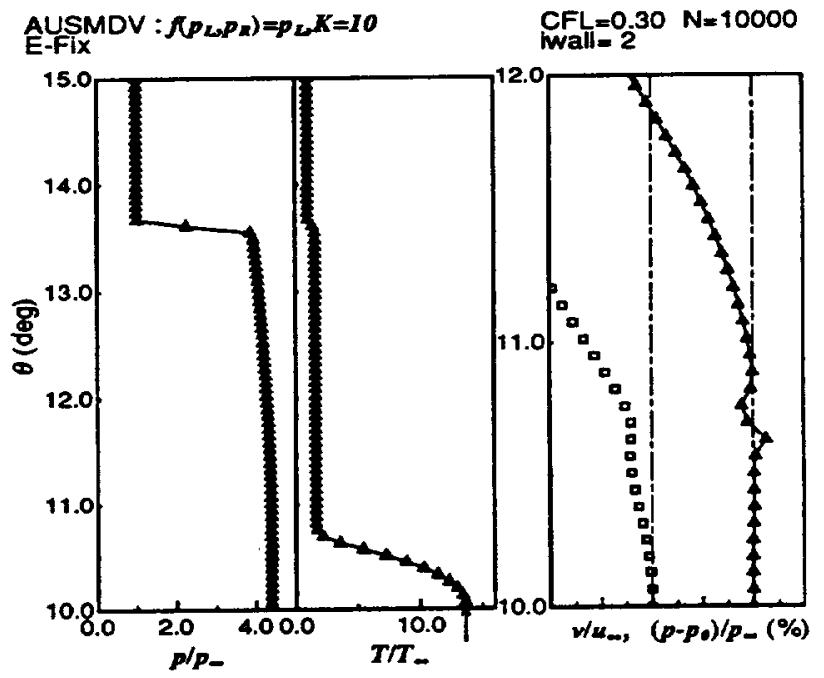

Fig. 14(e) Conical flow problem by second-order AUSMDV. 


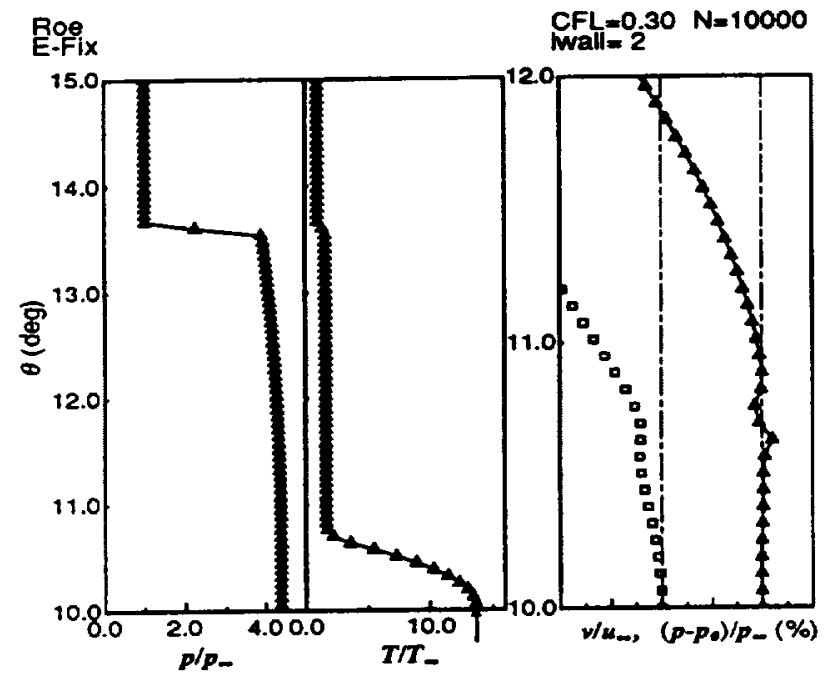

Fig. 14(f) Conical flow problem by second-order Roe scheme.

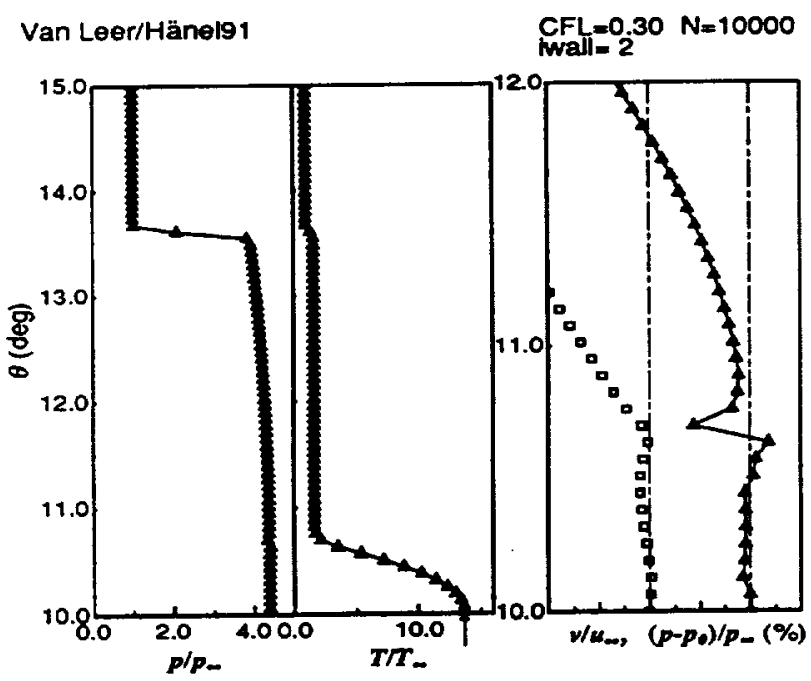

Fig. 14(g) Conical flow problem by second-order Van Leer FV/DS.

The second-order results by the nse of the MUSCLtype TVD scheme with minmod limiter, are shown in Figs. 14(e-g) for the AUSMDV, the Roe scheme and Van Leer's FV/DS, respectively. The solutions of the AUSMDV and the Roe scheme are almost identical while the kink of Van Leer's FV/DS still remains although it is improved.

\subsection{Hypersonic Flow around a Blunt Body}

Inviscid and viscous hypersonic flows around a cylinder were solved for the ideal gas. First the inviscid flow is presented at the free-stream Mach number of 6 with a $45 \times 41$ grid system and mirror wall condition being used. This grid system includes control volumes along the stagnation streamline. Figures $15(\mathrm{a}-\mathrm{d})$ show the results by the AUSMDV, the Godunov scheme, the Roe scheme and the Osher scheme, respectively. The density contours as well as the profiles of pressure, temperature and density along the stagnation stream line are shown. The AUSMDV clearly captures a strong shock wave with no overshoot. The Roe scheme shows a serious carbuncle phenomenon, and the Osher scheme has a singularity in the density/temperature profile even though the pressure is correctly solved. It is noted that when the control volumes were off stagnation stream line, the Osher scheme did not suffer from this phenomenon. The Godunov scheme is acceptable, however the density, which should increase by adiabatic compression along the stagnation stream line, does not have a monotonic profile. The proposed shock-fix was applied to the Roe scheme, and the result is shown in Figure 15(e). The carbuncle phenomenon is now cured, and the solution is now similar to that of the Godunov scheme. But the density does not take the maximum value at the stagnation point. This situation is improved when a higherorder scheme is used. Figure 15(f) shows the result, in which the non-MUSCL fully-upwind second-order TVD scheme[5] was used. The non-conservation of enthalpy of the Roe scheme is compensated for by higher-order accuracy. On the other hand, the AUSMDV conserves the total enthalpy for the steady state, so it makes the stagnation temperature exact, and gives a monotonic density profile.

The full Navier-Stokes equations were solved using the same grid at $R e=1000$, which results in a high cell Reynolds number of 25 at the wall. The wall temperature was fixed at the free-stream temperature, and the no-slip boundary condition was used. The density on the wall was calculated by assuming no mass fux from the wall. Figures 16(a)-(d) show the solution of the the AUSMDV, Roe's FDS, Van Leer's FVS and Van Leer's FV/DS, respectively. These figures show the contours of pressure and temperature, the profile of pressure and temperature along the stagnation stream line, and Stanton number on the cylinder surface. The solution by the AUSMDV in Fig. 16(a) is similar to that of the Roe scheme with shock-fix in Fig. 16(b). It is noticeable that the AUSMDV predicts a slightly higher Stanton number(heat flux) than that of the Roe scheme, indicating less amount of numerical dissipation in the AUSMDV result. Figure $16(\mathrm{c})$ is a solution by Van Leer's FVS, which produces a thick boundary layer, predicting much reduced heat flux. Figure $16(\mathrm{~d})$ is a solution by Van Leer's FV/DS, and exhibits a kink in the pressure field near the wall although the resolution of the boundary layer is improved. The same problem was solved on a finer grid( $149 \times 62$, cell Reynolds number of 4 at the wall) by the use of the AUSMDV and the Roe scheme. The results are shown in Fig. $16(\mathrm{e})$ and $16(\mathrm{f})$, where both schemes predict almost the same heat flux. This grid dependency study indicates that the AUSMDV is capable of evaluating heat flux as accurately as Roe's approximate Riemann solver. 


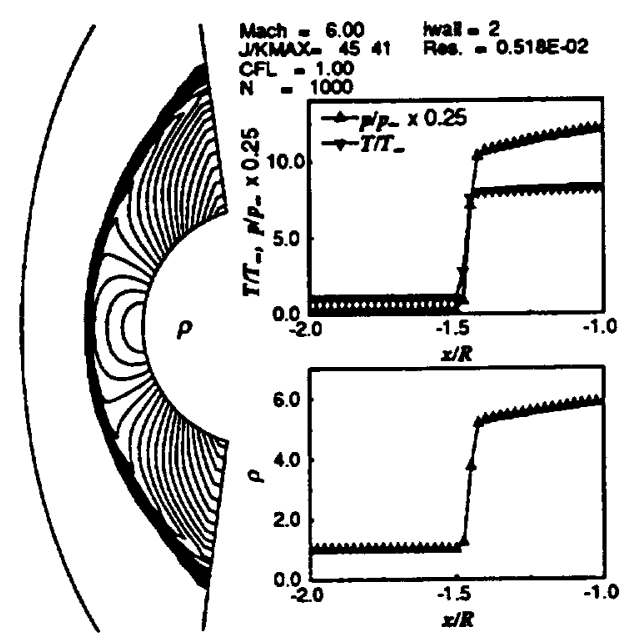

Fig. 15(a) Hypersonic flow around a cylinder by AUSMDV.

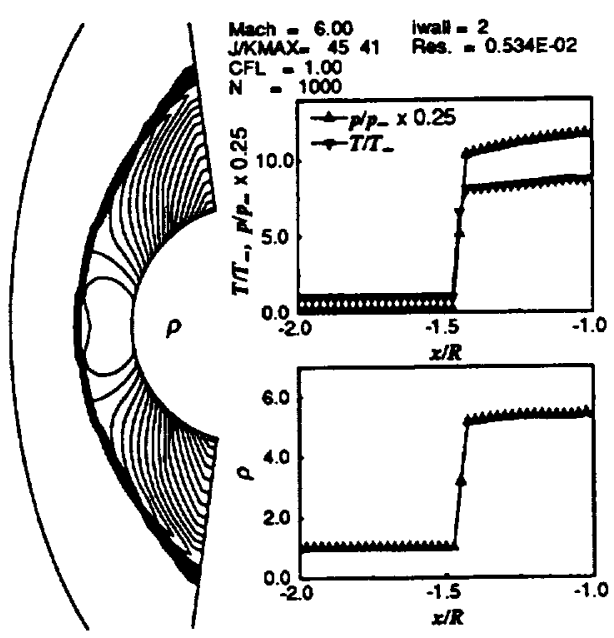

Fig. 15(b) Hypersonic flow around a cylinder by Godunov scheme.

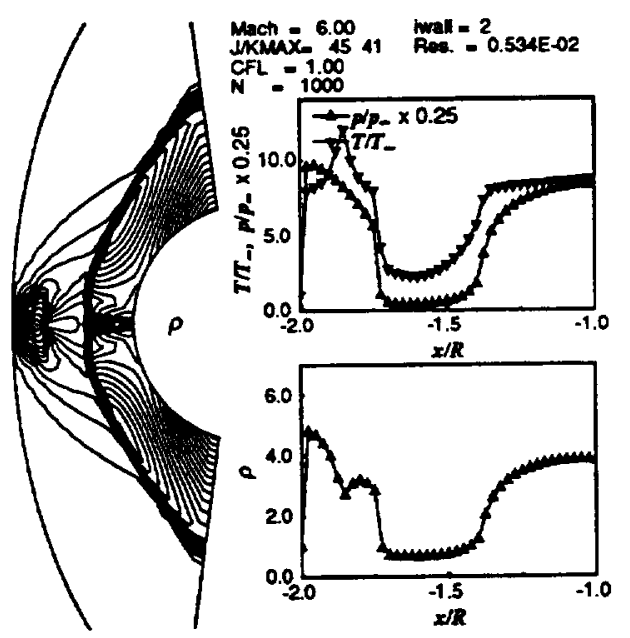

Fig. 15(c) Hypersonic flow around a cylinder by Roe scheme.

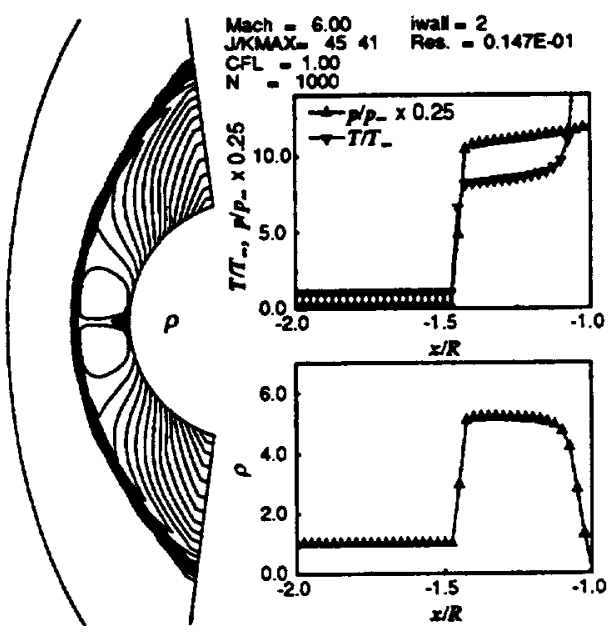

Fig. 15(d) Hypersonic flow around a cylinder by Osher scheme.

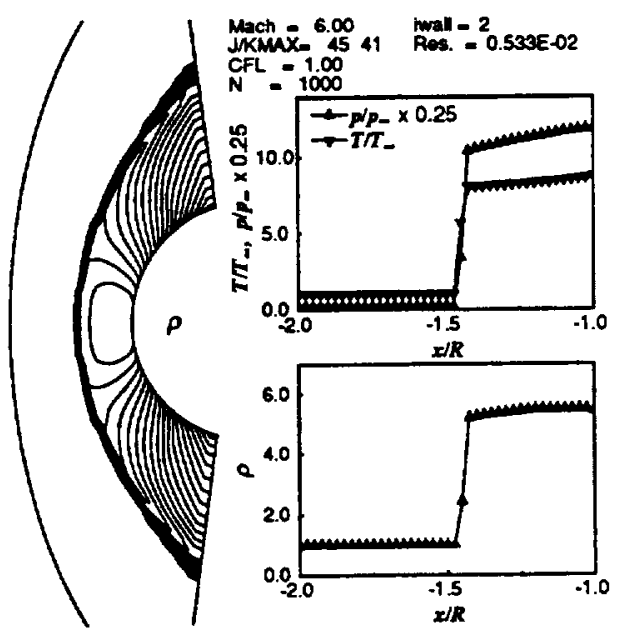

Fig. 15(e) Hypersonic flow around a cylinder by Roe scheme with shock-fix.

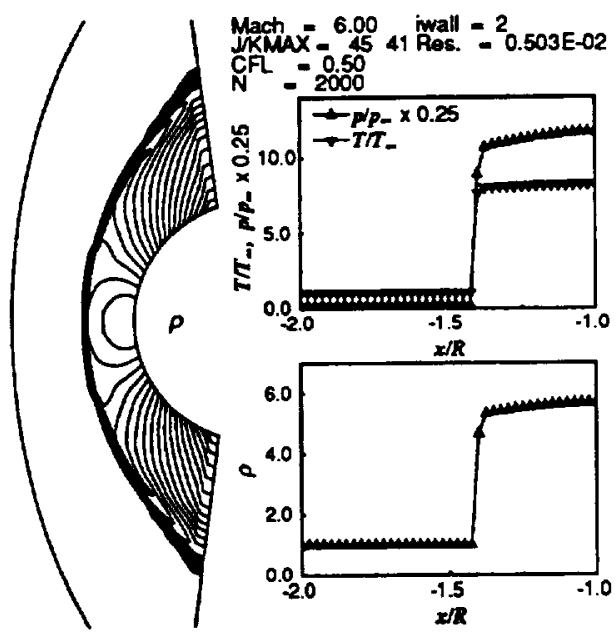

Fig. 15(f) Hypersonic flow around a cylinder by second-order Roe scheme with shock-fix. 

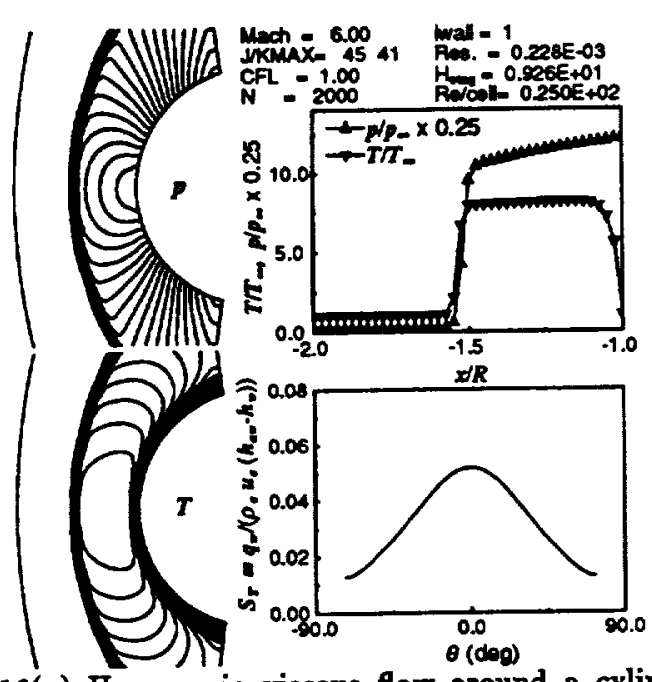

Fig. 16(a) Hypersonic viscous flow around a cylinder by AUSMDV.

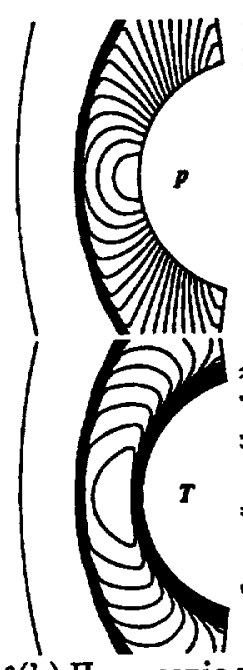

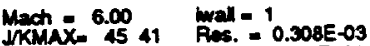


Fig. 16(b) Hypersonic viscous flow around a cylinder by Roe scheme.
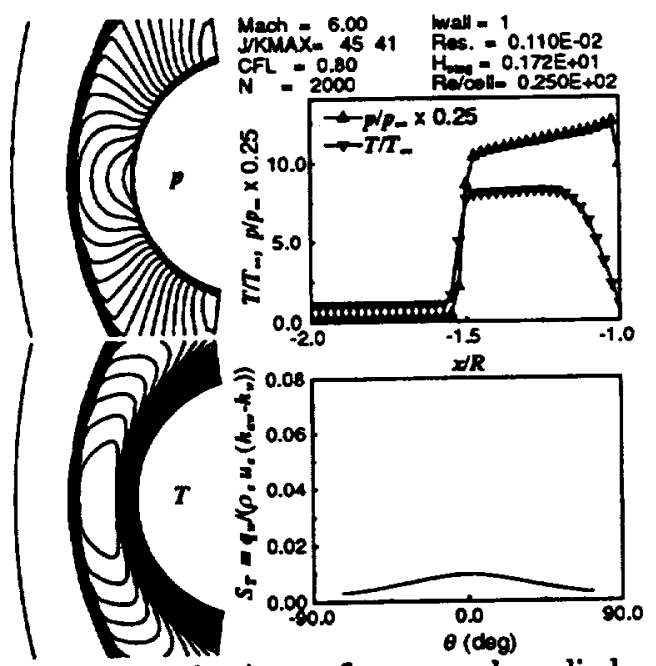

Fig. 16(c) Hypersonic viscous flow around a cylinder by Van Leer's FVS.


Fig. 16(d) Hypersonic viscous flow around a cylinder by Van Leer's FV/DS.

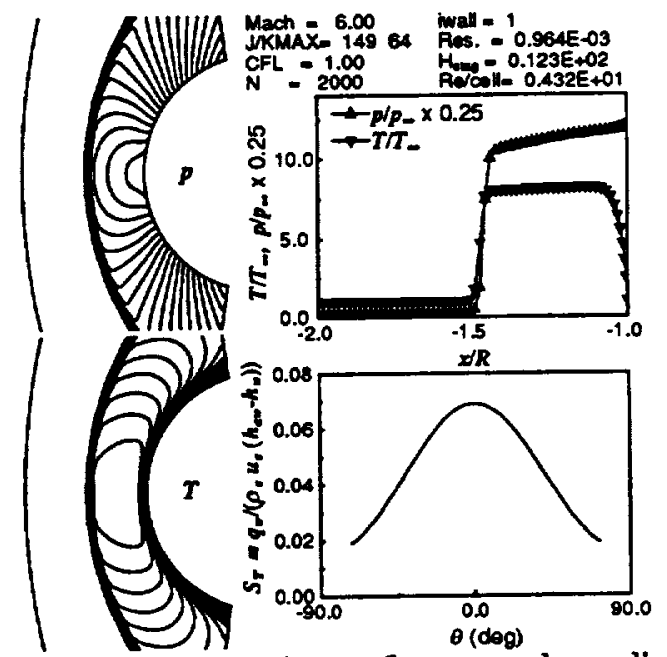

Fig. 16(e) Hypersonic viscous flow around a cylinder by AUSMDV with a fine grid system.

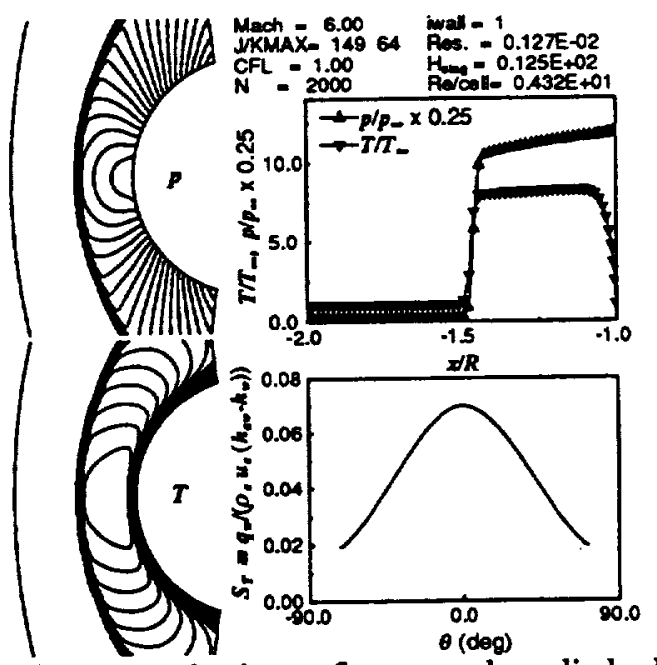

Fig. 16(f) Hypersonic viscous flow around a cylinder by Roe scheme with a fine grid system. 
4.5 Hypersonic Flow with the Two-Temperature Model

The AUSMDV was applied to a thermal-chemical nonequilibrium flow: a hypersonic flow around a cylinder with diameter of $1 \mathrm{~m}$. The cylinder is traveling at $7 \mathrm{~km} / \mathrm{sec}$ (Mach 25.3) at an altitude of 85km. Park's twotemperature model and Blottner's 7-species chemical reaction model were used with a grid system of $32 \times 50$. Steady state solutions were obtained with the residual being reduced about three-order of magnitude by the point implicit time integration method. The inviscid flow solution is shown in Fig. 17, which includes the contours of pressure, translational-rotational temperature and vibrational-electronic temperature along with profiles of these quantities as well as molar concentrations on the stagnation stream line. The AUSMDV can capture a strong shock wave crisply with no oscillation in the field of the pressure, translational/vibrational temperature and chemical species concentrations.

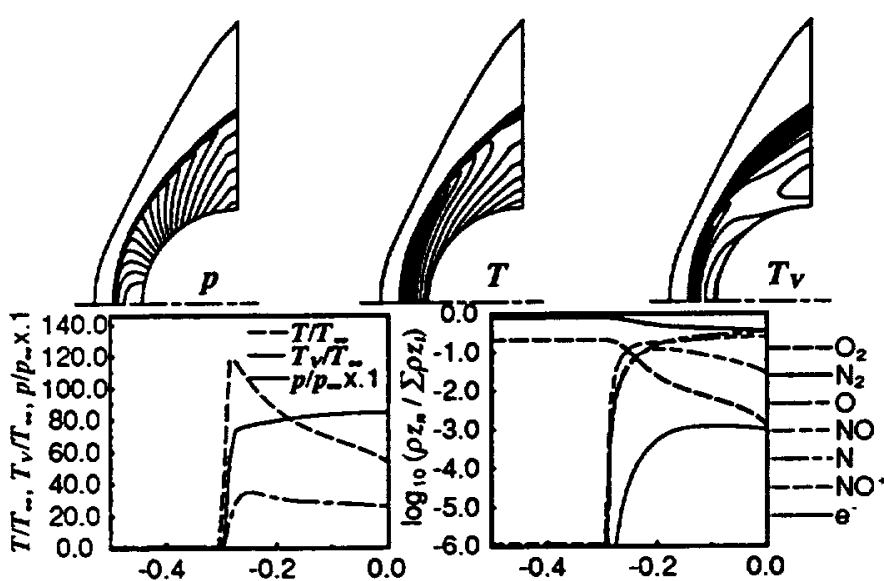

Fig. 17 Inviscid hypersonic flow with the two-temperature model around a cylinder by AUSMDV.

The viscous flow was solved using the same flow condition and grid, although this grid is very coarse for viscous computation with the cell Reynolds number near the wall being about 50. Figures 18(a), 18(b) and $18(\mathrm{c})$ are results by the AUSMDV, the generalized Roe scheme[37] with shock-fix and the Hānel FVS, respectively. The AUSMDV and the Roe scheme give similar solutions, while the Hänel FVS shows a very thick boundary layer due to the numerical dissipation. The heat flux at the stagnation was $78.9 \mathrm{KW} / \mathrm{m}^{2}$, $76.2 \mathrm{KW} / \mathrm{m}^{2}, 13.6 \mathrm{KW} / \mathrm{m}^{2}$ for the AUSMDV, the Roe FDS and the Hänel FVS, respectively. This again confirms that the AUSMDV is as accurate as the Roe scheme in evaluating heat flux. It is noted that, especially in the nonequilibrium flow computations, the AUSMDV is much simpler and more efficient than the Roe-type scheme, because the latter needs the information of various physical quantities in order to construct a Roe-averaged Jacobian matrix [37, 38].

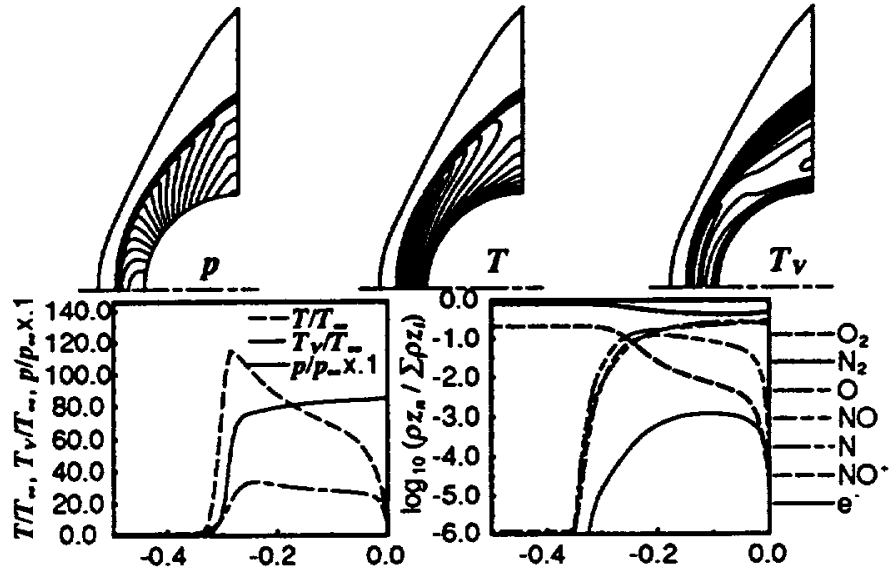

Fig. 18(a) Viscous hypersonic flow with the two-temperature model around a cylinder by AUSMDV.

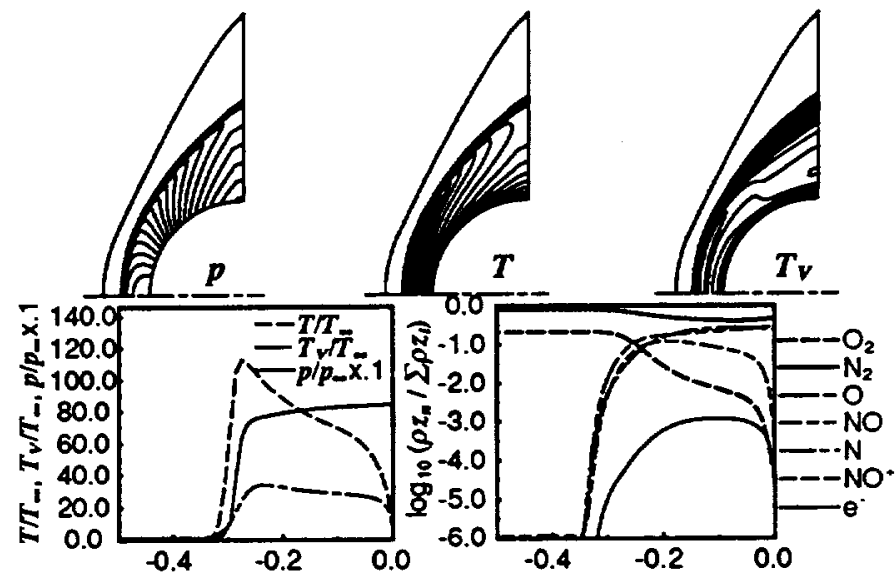

Fig. 18(b) Viscous hypersonic flow with the two-temperature model around a cylinder by Roe scheme.



Fig. 18(c) Viscous hypersonic flow with the two-temperature model around a cylinder by Hanel FVS. 


\section{CONCLUSION}

In this paper we have proposed a flux splitting scheme which is equipped with favorable properties: high-resolution for contact discontinuities; conservation of enthalpy for steady flows; numerical efficiency; applicability to chemically reacting flows. The results of varjous numerical experiments including that of a thermochemical nonequilibrium flow indicate the soundness of the proposed scheme. In addition, a shock-fix is presented, which cures the carbuncle phenomenon and is applicable to the Roe scheme as well as to the new scheme presented here.

\section{ACKNOWLEDGMENT}

We would like to acknowledge Dr. Van Der Vegt for a fruitful discussion about the Osher scheme and the entropy-fix. Also thanks go to Dr. Obayashi for the discussion about the cure for the carbuncle phenomenon.

\section{REFERENCES}

[1] Van Leer, B., "Towards the Ultimate Conservative Difference Scheme V. A Second-Order Sequel to Godunov's Method," J. Comput. Phys., vol.32, 1979, pp.101-136.

[2] Colella, P. and Woodward, P.R., "The Piecewise Parabolic Method (PPM) for Gas-Dynamic Simulations, ${ }^{n}$ J. Comput. Phys., vol.54, 1984, pp.249331.

[3] Harten, A., "High Resolution Schemes for Hyperbolic Conservation Laws," J. Comput. Phys., vol.49, 1983, pp.357-393.

[4] Yee, H.C., "Linearized Form of Implicit TVD Schemes for Multi-Dimensional Euler and NavierStokes Equations," Computers and Mathematics with Applications, vol.12A, 1986, pp.413-432.

[5] Chakravarthy, S.R. and Osher, S., "A New Class of High Accuracy TVD Schemes for Hyperbolic Conservation Laws," AIAA Paper 85-0363, January 1985.

[6] Chakravarthy, S.R., "The Versatility and Reliability of Euler Solvers Based on High-Accuracy TVD Formulations," AIAA Paper 86-0243, Janvary 1986.

[7] Anderson, W.K., Thomas, J.L. and Van Leer, B., "Comparison of Finite Volume Flux Vector Splittings for the Euler Equations," AIAA Journal, vol.24, 1986, pp.1453-1460; also AIAA Paper 850122 , January 1985.
[8] Lion, M.-S., "A Generalized Procedure for Constructing an Upwind-Based TVD Scheme," AIAA Paper 87-0355, January 1987.

[9] Takakura,Y., Ishiguro,T. and Ogawa,S., "On the Recent Difference Schemes for the ThreeDimensional Euler Equations," AIAA Paper 871151CP, June 1987.

[10] Godunov, S.K., "A Finite Difference Method for the Numerical Computation of Discontinuous Solutions of the Equations of Fluid Dynamics," Mat. Sb. vol.47, 1959, pp.357-393.

[11] Roe, P.L., "Approximate Riemann Solvers, Parameter Vectors, and Difference Schemes," J. Comput. Phys., vol.43, 1981, pp.357-372.

[12] Quirk, J.J., "A contribution to the Great Riemann Solver Debate," ICASE Report 92-64, 1992.

[13] Harten, A., Lax, P.D. and Van Leer, B., "On Upstream Differencing and Godunov-Type Schemes for Hyperbolic Conservation Laws," SIAM Review, vol.25, 1983, pp.35-61.

[14] Einfeldt, B., "On Godunov-Type Methods for GasDynamics," SIAM J. Numer. Anal., vol.25, 1988, pp.357-393.

[15] Einfeldt, B., Munz, C.C., Roe, P.L. and Sjogreen, B., "On Godunov-Type Methods near Low Densities," J. Comput. Phys., vol.92, 1991, pp.273295.

[16] Wada, Y., "On the Godunov-Type Schemes - An Improvement of HLLEM Scheme and its Extension to Chemically Reacting Flows," NAL TR-1189, 1993(in Japanese).

[17] Obayashi,S. and Wada,Y., "Practical Formulation of a Positively Conservative Scheme, ${ }^{n}$ to appear in Notes in AIAA Journal.

[18] Roberts, T.W., “The Behavior of Flux Difference Splitting Schemes near Slowly Moving Shock Waves," J. Comput. Phys., vol.90, 1990, Pp.141160.

[19] Osher, S. and Solomon, F., "Upwinded Schemes for Hyperbolic Systems of Conservation Laws," Math. Comput., vol.38, 1981, pp.339-377.

[20] Steger, J.L. and Warming, R.F., "Flux Vector Splitting of the Inviscid Gasdynamic Equations with Application to Finite-Difference Methods," J. Comput. Phys., vol.40, 1981, pp.263-293. 
[21] Van Leer, B., "Flux-Vector Splitting for the Euler Equations," Lecture Notes in Physics, vol.170, 1982, pp.507-512.

[22] Hänel, D., Schwane, R., and Seider, G., "On the Accuracy of Upwind Schemes for the Solution of the Navier-Stokes Equations," AIAA Paper 87$1105 \mathrm{CP}, 1987$.

[23] Liou, M.-S. and Steffen, C.J., “High-Order Polynomial Expansions (HOPE) for Flux-Vector Splitting," NASA TM-104452, 1991.

[24] Hänel, D. and Schwane, R., "An Implicit FluxVector Splitting Scheme for the Computation of Viscous Hypersonic Flow," AIAA Paper 89-0274, January 1989.

[25] Van Leer, B., "Flux-Vector Splitting for the 1990s," NASA CP-3078, 1991, pp.203-214.

[26] Liou, M.-S. and Steffen,C.J., "A New Flux Splitting Scheme," NASA TM104404, 1991; also in $J$. Comput. Phys. vol.107, 1993, pp. 23-39.

[27] Liou, M.-S., "On a New Class of Flux Splittings," Lecture Notes in Physics, vol.414, 1993, pp.115119.

[28] Roe, P.L., "A Survey of Upwind Differencing Techniques," Workshop on Some Recent Trends in Computational Fluid Dynamics, NASA Ames RC, August, 1988.

[29] Park, C., "Assessment of a Two-Temperature Kinetic Model for Dissociating and Weakly Ionizing Nitrogen," AIA A Journal of Thermophysics, vol.2, 1988, pp.8-16.

[30] Millikan, R.C. and Johnson, R.E., "Systematics of Vibrational Relaxation," J. Chem. Phys., vol.39, 1963, pp.3209-3213.

[31] Wada, Y., Ogawa, S and Kubota, H., "On the Thermo-Chemical Models for Hypersonic Flows," 4th ISCFD, Davis, 1991, pp.719-728, also in Computers and Fluids, vol.22, 1993, pp.179-187.

[32] Gnoffo, P.A., Gupta, R.N. and Shinn, J.L., "Conservation Equations and Physical Models for Hypersonic Air Flows in Thermal and Chemical Nonequilibrium," NASA TP-2867, 1989.

[33] Blottner, F.C.,Johonson, M. and Ellis, M., "Chemically Reacting Viscous Flow Program for MultiComponent Gas Mixtures," Report No. SC-RR-70 754, Sandia Laboratories, Albuquerque, New Mexico, Dec. 1971.
[34] Chskravarthy, S.R., and Osher, S., "Computing with High-Resolution Upwind Schemes for Hyperbolic Equations," Lectures in Applied Mathematics, vol.22., 1985.

[35] Roe, P.L., "Sonic Flux Formulae," SIAM J. Stat. Comput., vol.13, 1992, pp.611-630.

[36] Van Leer, B., Thomas, J.L., Roe, P.L., Newsome, R.W., "A Comparison of Numerical Flux Formulas for the Euler and Navier-Stokes Equations," AIAA paper $87-1104 \mathrm{CP}, 1987$.

[37] Liu, Y., and Vinokur, M., "Nonequilibrium Flow Computations I. An Analysis of Numerical Formulations of Conservation Laws," J. Comput. Phys., vol.83, 1989, pp.373-397.

[38] Shuen, J.-S., Liou, M.-S. and Van Leer, B., "Inviscid Flux-Splitting Algorithms for Real Gases with Non-equilibrium Chemistry," J. Comput. Phys., vol.90, 1990, pp.371-395. 
Public reporting burden for this collection of information is estimated to average 1 hour per response, including the time for reviewing instructions, searching existing data sources, Pertion of intormation. Send comments regarding this burden estimate or any other aspect of this

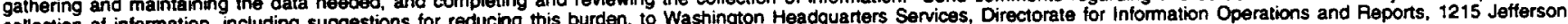
collection of in

\begin{tabular}{|l|c|l|}
\hline 1. AGENCY USE ONLY (Leave blank) & $\begin{array}{c}\text { 2. REPORT DATE } \\
\text { January } 1994\end{array}$ & $\begin{array}{c}\text { 3. REPORT TYPE AND DATES COVERED } \\
\text { Technical Memorandum }\end{array}$
\end{tabular}

\section{TITLE AND SUBTITLE}

A Flux Splitting Scheme With High-Resolution and Robustness for Discontinuities

6. AUTHOR(S)

Yasuhiro Wada and Meng-Sing Liou
WU-505-90-5K

PERFORMING ORGANIZATION REPORT NUMBER

E-8311

National Aeronautics and Space Administration

Lewis Research Center

Cleveland, Ohio 44135-3191

9. SPONSORINGMONITOAING AGENCY NAME(S) AND ADDRESS(ES)

10. SPONSORINGMONITORING AGENCY REPORT NUMBER

National Aeronautics and Space Administration

NASA TM-106452

Washington, D.C. 20546-0001

ICOMP-93-50

ALAA-94-0083

11. SUPPLEMENTARY NOTES

Prepared for the 32nd Aerospace Sciences Meeting and Exhibit sponsored by the American Institute of Aeronautics and Astronautics, Reno, Nevada, January 10-13, 1994. Yasuhiro Wada, Institute for Computational Mechanics in Propulsion, NASA Lewis Research Center (work funded by NASA Cooperative Agreement NCC3-233); and Meng-Sing Liou, NASA Lewis Research Center. ICOMP Program Director, Louis A. Povinelli, (216) $433-5818$

12a. DISTRIBUTION/AVAILABILITY STATEMENT

Unclassified - Unlimited

Subject Categories 34 and 64

13. ABSTRACT (Maximum 200 words)

A flux splitting scheme is proposed for the general non-equilibrium flow equations with an aim at removing numerical dissipation of Van-Leer-type flux-vector splittings on a contact discontinuity. The scheme obtained is also recognized as an improved Advection Upwind Splitting Method (AUSM) where a slight numerical overshoot immediately behind the shock is eliminated. The proposed scheme has favorable properties: high-resolution for contact discontinuities; conservation of enthalpy for steady flows; numerical efficiency; applicability to chemically reacting flows. In fact, for a single contact discontinuity, even if it is moving, this scheme gives the numerical flux of the exact solution of the Riemann problem. Various numerical experiments including that of a thermo-chemical nonequilibrium flow were performed, which indicate no oscillation and robustness of the scheme for shock/expansion waves. A cure for carbuncle phenomenon is discussed as well.

\section{SUBJECT TERMS}

Flux-splitting; Compressible; Chemically reacting flow; Upwinding

\begin{tabular}{|c|c|c|}
\hline $\begin{array}{c}\text { 17. SECURITY CLASSIFICATION } \\
\text { OF REPORT } \\
\text { Unclassified }\end{array}$ & $\begin{array}{c}\text { 18. SECURITY CLASSIFICATION } \\
\text { OF THIS PAGE } \\
\text { Unclassified }\end{array}$ & $\begin{array}{c}\text { 19. SECURITY CLASSIFICATION } \\
\text { OF ABSTRACT } \\
\text { Unclassified }\end{array}$
\end{tabular}

Standard Form 298 (Rev. 2-89)

Prescribed by ANSI Std. 239-18 298-102 